\title{
The ISAD (Infraslow Neurofeedback for Anxiety and Depression) Study: A Protocol for a Trans- Diagnostic, Randomized, Double-Blind, Sham- Controlled, Dose-Response, Parallel-Group Trial
}

\section{Tyson Perez ( $\nabla$ drtysondc@gmail.com )}

University of Otago https://orcid.org/0000-0003-3054-5928

\section{Paul Glue}

University of Otago - Dunedin Campus: University of Otago

\section{Divya B Adhia}

University of Otago - Dunedin Campus: University of Otago

Mark Smith

NONE

\section{Dirk De Ridder}

University of Otago - Dunedin Campus: University of Otago

Jiaxu Zeng

University of Otago

Peter Dillingham

University of Otago

Imran Niazi

New Zealand College of Chiropractic

\section{Study protocol}

Keywords: Internalizing disorders, Generalized anxiety disorder, Major depressive disorder, Social anxiety disorder, EEG, Neurofeedback, Infraslow, Triple network, Salience network, Default mode network

Posted Date: June 14th, 2021

DOI: https://doi.org/10.21203/rs.3.rs-173667/v1

License: (c) (1) This work is licensed under a Creative Commons Attribution 4.0 International License. Read Full License 


\section{Abstract}

Background: The core intrinsic connectivity networks (ICNs), encompassing the default-mode network (DMN), salience network (SN) and central executive network (CEN), have been shown to be dysfunctional in individual's with internalizing disorders (IDs; e.g. major depressive disorder, MDD; generalized anxiety disorder, GAD; social anxiety disorder, SOC). As such, source-localised electroencephalogram neurofeedback (EEG-NFB) therapy targeting key cortical nodes within these networks has the potential to reduce symptoms associated with IDs and restore normal core ICN function. We intend to conduct a transdiagnostic, randomized, double-blind, sham-controlled, dose-response, parallel-group trial of standardized low-resolution electromagnetic tomography electrophysiological infraslow $(<0.1 \mathrm{~Hz})$ fluctuation neurofeedback (sLORETA elSF-NFB) 3 times per week over 4 weeks in participants $(n=60)$ with IDs. Our primary aim will be to assess the clinical efficacy of SLORETA eISF-NFB via relevant patientreported outcomes (PROs).

Methods: We will randomly assign participants with a current diagnosis of MDD, GAD, and/or SOC to one of four groups: 1) 12 sessions of posterior cingulate cortex (PCC) up-training $(n=15), 2) 6$ sessions of yoked-sham training followed by 6 sessions of PCC up-training $(n=15), 3) 12$ sessions of concurrent midcingulate (MCC) down-training and PCC up-training ( $n=15)$, or 4) 6 sessions of yoked-sham training followed by 6 sessions of concurrent MCC down-training and PCC up-training. Transdiagnostic PROs, as well as resting-state neuro-physiological measures (EEG; electrocardiography, ECG; electrodermal activity, EDA), will be collected from all subjects at baseline, mid-training, 1 week post-training, and 1 month posttraining. We will further compare baseline PROs and neuro-physiological measures to age- and sexmatched non-ID (i.e. no ID diagnosis) controls.

Discussion: This protocol will outline the rationale and research methodology for a clinical trial of sLORETA elSF-NFB targeting key nodes within the core ICNs in a population with IDs with the primary aim being to assess its specific (e.g. non-placebo induced) efficacy via PROs.

Trial Registration: Our study was prospectively registered with the Australia New Zealand Clinical Trials Registry (ANZCTR; Trial Id: ACTRN12619001428156)

\section{Administrative Information}

Note: the numbers in curly brackets in this protocol refer to SPIRIT checklist item numbers. The order of the items has been modified to group similar items (see http://www.equator-network.org/reportingguidelines/spirit-2013-statement-defining-standard-protocol-items-for-clinical-trials/). 
Title $\{1\}$

The ISAD (InfraSlow neurofeedback for Anxiety and Depression) study: a protocol for a trans-diagnostic, randomized, double-blind, sham-controlled, dose-response, parallel-group, trial

Trial registration $\{2 a \quad$ Australia New Zealand Clinical Trials Registry: and $2 b$ \}

ACTRN12619001428156

Protocol version $\{3\} \quad$ Version 2: 15 May, 2020

Funding $\{4\}$

This clinical trial is funded by the Department of Surgical Sciences, University of Otago, Dunedin, New Zealand.

Author details $\{5 a\}$

Tyson Michael Perez ${ }^{1}$, Paul Glue ${ }^{2}$, Divya B Adhia ${ }^{3}$, Mark Smith ${ }^{4}$, Dirk De Ridder $^{5}$

- 1 Departments of Surgical Sciences \& Psychological Medicine, University of Otago, Dunedin, New Zealand. Email: tyson.perez@postgrad.otago.ac.nz

- 2 Department of Psychological Medicine, University of Otago, Dunedin, New Zealand. Email: paul.glue@otago.ac.nz

- ${ }^{3}$ Department of Surgical Sciences, University of Otago, Dunedin, New Zealand. Email: divya.adhia@otago.ac.nz

- ${ }^{4}$ Neurofeedback Therapy Services of New York, New York, USA. Email: mark@neurofeedbackservicesny.com

- ${ }^{5}$ Department of Surgical Sciences, University of Otago, Dunedin, New Zealand. Email: dirk.deridder@otago.ac.nz

Corresponding author: Tyson M Perez, tyson.perez@postgrad.otago.ac.nz

Name and contact information for the This clinical trial is sponsored by the Department of Surgical Sciences, trial sponsor $\{5 b\}$

Role of sponsor $\{5 \mathrm{c}\}$

The funder/sponsor had no role in the design, implementation, analyses, interpretation, or dissemination of the results.

\section{Introduction}

\section{Background and rationale $\{6 a\}$}

Mental disorders are one of the most common causes of morbidity and mortality worldwide(1) with rates markedly increasing in recent years(2-6). Here in New Zealand, it is estimated that one in five is dealing with a mental illness at any given time with a majority likely to experience at least one episode at some point in their lifetime(7). Internalising disorders (IDs), which include all anxiety (e.g. generalised anxiety disorder, GAD; social phobia, SOC) and depressive (e.g. major depressive disorder, MDD) disorders, are the most common mental illnesses characterised by neuroticism and distress experienced inwardly $(8,9)$. 
Females(10-13) and young people (i.e. <65 years) $(12,14-24)$ appear to be disproportionately affected by IDs. Unfortunately, there are numerous shortcomings of traditional frontline therapies (i.e. pharmacotherapy and psychotherapy) including high failure rates(25-31), lack of access (12, 32-35), and marked adverse side-effects(32, 33, 36-39).

In recent years, neuropsychiatric research is pointing to trans-diagnostic, neurobiological aberrations specifically involving the so-called 'core' intrinsic connectivity networks (ICNs) which include the default mode network (DMN), central executive network (CEN) and salience network (SN)(40-43). Briefly, the $\mathrm{DMN}$ is anchored in the posterior cingulate cortex (PCC), medial prefrontal cortex (mPFC) and hippocampus, and is generally believed to subserve internally-directed thought $(44,45)$. The CEN is associate with executive functioning and putatively anchored in the dorsolateral prefrontal cortex (dIPFC) and posterior parietal cortices (PPC)(46-52). Lastly, the SN is anchored in the anterior insula (aINS), midcingulate cortex (MCC) and amygdala, and is believed to be important for the detection of salient stimuli and switching between the other core-ICNs $(53,54)$. Additionally, the core-ICNs have also been associated with autonomic nervous system (ANS) modulation(55-60). This latter finding is intriguing considering that ANS irregularities are a consistent finding across psychopathologies $(39,61)$. One of the most robust ANS disturbances found in IDs is cardiac dysautonomia in the form of reduced $\operatorname{HRV}(29,36$, $39,61-94)$ which has significant clinical implications considering that cardiovascular disease is the leading cause of mortality in people with mental illness $(39,95,96)$. Similarly, electrodermal activity (EDA), a sympathetic marker, is consistently found to be disturbed across IDs(97-103).

In 2011, the converging neurobiological evidence led Menon and colleagues to propose a unifying theory of psychopathology termed the 'triple network model'(41, 104-106). The central tenet of this theory is that the sensorial, cognitive, affective, and behavioural dysfunctions associated with mental illnesses are the result of disruptions within and between the core ICNs. Since its inception, support for this model has been rapidly mounting within the ID-domain (e.g. (107), (108), (109)and (110). Interestingly, a common finding within this model appears to be DMN-SN hyperconnectivity $(41,111-113)$. Notably, to our knowledge only one EEG-based trial have ever validated this triple network model in an ID population(114), however, their investigation was based entirely within traditional bandwidths (i.e. delta and above) thereby omitting electrophysiological infraslow fluctuations (elSFs; $<0.1 \mathrm{~Hz}$ ).

Standard clinical EEGs typically limit the recording bandwidth to traditional frequency bands (i.e. delta 1-4 Hz, theta $\sim 4-8 \mathrm{~Hz}$, alpha $\sim 8-12 \mathrm{~Hz}$, beta $\sim 12-30 \mathrm{~Hz}$, gamma $>30 \mathrm{~Hz}$ ) with electrophysiological infraslow fluctuations (elSFs; $<0.1 \mathrm{~Hz}$ ) filtered out(115). elSFs were first reported in the by Russian scientists over half a century ago, first in rabbits $(116,117)$ and shortly thereafter in humans $(118)$, but have received little attention until recently due, in part, to technological challenges(115, 119-122). Putatively engendered by a combination of neuronal and glial currents $(115,120,123-128)$, elSFs have been shown in both cortical $(117,118,129)$ and subcortical $(128,130-134)$ tissues and are believed to coordinate large-scale ICN organisation and information exchange $(120,135-143)$. Taken together, it could be hypothesised that treatments specifically targeting core-ICN elSFs may offer clinical utility in the treatment of IDs. 
EEG-neurofeedback (EEG-NFB) is a non-invasive therapy aimed at modulating brain function by teaching individuals, via operant conditioning, to self-regulate their brain function via auditory, visual, and/or tactile feedback(144). EEG-NFB has shown significant positive clinical effects in a wide various of conditions(145-150) including IDs(35, 104, 114, 151-180). Intriguingly, efficacy has been shown to continue to improve following treatment cessation putatively due to neuroplastic effects(181). The general lack of extended follow-up and failure to assess for the potential emergence of delayed treatment effects has been a criticism levelled against prior NFB trials(181). That said, some researchers assert that NFB's efficacy rests almost entirely on 'non-specific' psychosocial factors (e.g. expectations, motivation, demand characteristics, context)(182-192). Proponents contend, however, assessment of EEG-learning (i.e. ability to regulate the targeted electrophysiological parameter) is essential in order to properly evaluate the specific efficacy of NFB(309-312) and demonstration of this learning was conspicuously absent in the trials touted by skeptics as evidence of wholly non-specific effects(312-314).

Advanced source-localization (i.e. standardized low resolution brain electromagnetic tomography; sLORETA)(193) combined with EEG-NFB targeting elSFs (sLORETA elSF-NFB) is novel introduction to the EEG-NFB field that has been shown by our research group in a feasibility trial to improve, based upon patient reported outcomes (PROs), sleep and wellbeing with minimal side-effects in an obese female population $(194,195)$. Additionally, it was shown to modulate ISFs within the targeted region-of-interest (ROI)(194). To our knowledge, this is the first RCT examining the clinical efficacy of sLORETA elSF-NFB for IDs.

\section{Objectives $\{7\}$}

The primary objectives of the current study are to assess the clinical efficacy of 1) active versus sham sLORETA eISF-NFB. 2) 6 versus 12 active sLORETA eISF-NFB sessions, 3) single- versus multi-region modulation, and 4) short vs medium term follow-up (i.e. post 1-week vs post 1-month) in people with IDs via transdiagnostic PROs. Secondary objectives will be to assess the efficacy of sLORETA eISF-NFB in the modulation of activity/connectivity within and between core ICNs as well as markers of ANS function. Further, we want to characterize functional aberrations within and between the core-ICNs, particularly within the infraslow band $(0.01-0.1 \mathrm{~Hz})$, in an ID population relative to age- and sex-matched non-ID controls.

We hypothesize:

- sLORETA elSF-NFB will show specific clinical efficacy (i.e. active > sham) in an ID population via improvements in relevant transdiagnostic PRO measures as well neuro-physiological measures which will be augmented by 1 ) more treatment sessions (12 active sessions $>6$ active sessions), 2 ) multi-region modulation (MCC down-training/PCC up-training > PCC up-training), and 3) time (post 1month > post 1-week).

- Relative to non-ID controls, ID participants will demonstrate significant activity and connectivity aberrations within and between the core-ICNs in multiple frequency bands, including infraslow (0.01$0.1 \mathrm{~Hz}$ ), which will be normalized by sLORETA elSF-NFB. 
- EEG-learning will be evident following active, but not sham, sLORETA elSF-NFB

\section{Trial Design \{8\}}

The ISAD (InfraSlow neurofeedback for Anxiety and Depression) study is a trans-diagnostic, randomized, double-blind (subjects and raters), sham-controlled (yoked by sex and ID diagnosis), dose-response (6 versus 12 genuine treatment sessions), parallel-group (4-arm) superiority, pilot trial using a 1:1:1:1 allocation ratio with primary endpoints at mid-training (i.e. after 6 sessions), 1-week post-training and 1month post-training. Importantly, our trans-diagnostic approach heeds recent calls for a more pragmatic, ecologically-valid clinical research(196-206).

\section{Methods: Participants, Interventions, And Outcomes}

\section{Study setting $\{9\}$}

This study will be undertaken in the EEGs at the Departments of Surgical Sciences and Psychological Medicine, University of Otago, Dunedin, New Zealand.

\section{Eligibility Criteria $\{10\}$}

Inclusion criteria:

1. Adult (18-64 years) females with current GAD, SOC, and/or MDD per the MINI[1]. Females were selected because IDs are reported to be more prevalent among females compared to men in the general population(10-13). Further, this trio of IDs was selected due to their high probability of cooccurrence/comorbidity (12, 17, 21, 198-200, 204, 205, 207-234), high prevalences(23, 235-237), chronic courses(229, 238), overlapping symptoms $(33,200,203,220,226,227,229,239,240)$, and putative shared neurobiological (i.e. core ICN) aberrations $(41,108)$.

2. Naïve to neurofeedback therapy

Exclusion criteria:

1. Taking new medications or dosages of existing medications ( $<4$ weeks)

2. Short-acting benzodiazepines or other drugs (e.g. amphetamines) that may influence ANS activity

3. Undergoing intensive psychotherapy (e.g. cognitive behavioural therapy)

4. Externalizing disorders (e.g. antisocial personality disorder, alcohol/substance abuse disorder, ADHD)

5. Thought disorders (e.g. mania, bipolar disorder, any psychotic disorder)

6. High-risk of suicide

7. Pregnant

8. Pacemaker 


\section{Who will take informed consent? $\{26 \mathrm{~b}\}$}

At the initial meeting, a trained male doctoral student will 1) provide each potential participant with a paper copy of the participant information sheet written in English, 2) query if they have read and understood the document, 3) ask if they have any questions about the trial, and 4) request written informed consent from individuals willing to participate in the study. Participants will be informed that they may withdraw at any time without giving a reason and that all data collected up to the point of withdrawal may be used in the final analyses.

\section{Additional consent provisions for collection and use of participant data and biological specimens \{26b\}}

Not applicable: no biological specimens will be collected and all data is to be used solely in accordance with this trial.

\section{Interventions}

\section{Explanation for the choice of comparators $\{6 \mathrm{~b}\}$}

The non-ID control group, which does not receive any intervention, will only be assessed at baseline in order to ascertain differences from the ID population in baseline PROs and neuro-physiological measures. To mitigate against selection bias(241), the same inclusion and exclusion criteria apply to the non-ID controls with the exception an ID diagnosis (e.g. MDD, GAD, SOC).

In the clinical cohort, our choice of sham-controls allows us to elucidate any potential specific (e.g. nonplacebo) effects and addresses widespread concerns of generally weak methodological designs in NFB trials $(35,242-244)$. Further, the staggered start allows us to perform a dose-response assessment and assures all clinical participants receive some genuine sLORETA eISF-NFB thereby minimizing the potential of sham-trial associated participation barriers(245) as well as addressing any potential ethical concerns of sham-only allocations.

\section{Intervention descriptions $\{11 \mathrm{a}\}$}

Following baseline assessments, clinical participants will be randomised to one of 4-arms: 1) 12 sessions of PCC elSF up-training, 2) 6 sessions of yoked-sham followed by 6 sessions of PCC elSF up-training, 3) 12 sessions of concurrent PCC eISF up-training and MCC eISF down-training, 4) 6 sessions of yokedsham followed by 6 sessions of concurrent PCC elSF up-training and MCC elSF down-training. Training will commence within 1-week of baseline \#2.

To help reduce impedances, subjects were asked to arrive with non-braided, dry, clean (i.e. no conditioner, gels, pastes, sprays) hair. Participants will attend three 30-minute sessions per week, every other day, over 4 weeks (12 sessions in total). 19-channel sLORETA elSF-NFB training will be performed using a 21channel DC coupled amplifier produced by Brainmaster Inc. and the BrainAvatar software (version 4.7.5.844) in a quiet, cool $\left(\sim 15^{\circ} \mathrm{C}\right)$, dimly lit room by an unblinded male researcher with $>2$ years of 
experience in the administration of NFB. Participants will be seated in a comfortable chair and an appropriately sized Comby EEG cap will be placed on the participant's head. Using a blunt need and syringe, the scalp will be mildly abraded prior to the application of an electrolyte gel beneath each electrode. EEGs will be recorded with the silver/silver chloride $(\mathrm{Ag} / \mathrm{AgCl})$ electrodes positioned according to the International 10-20 system (i.e., Fp1, Fp2, F3, F4, C3, C4, P3, P4, 01, 02, F7, F8, T3, T4, T5, T6, Fz, $\mathrm{Cz}, \mathrm{Pz}$ ) using a linked mastoids reference and a ground electrode positioned centrally between, F3, Fp1, Fz and Fpz. It should be noted that the purpose of the cool room and scalp abrasion is to mitigate contamination of the EEG signal by electro-dermal (i.e. sweat gland) potentials which are known to mimic brain-derived ISFs $(115,121)$. The impedances of the active electrodes will be kept below $10 \mathrm{k} \Omega$ and a 50 $\mathrm{Hz}$ notch filter will be set. During the set-up, participants will be asked to complete the DESS on an IPad.

Immediately prior to each training period, a demonstration of motion/electromyography (EMG) artefact alerts will be performed with instructions to avoid eye/head/face movements in order to minimize this non-rewarding feedback. Participants will then be instructed to close their eyes, relax, stay awake, and listen to the sound being played. They will be informed that the sound they hear reflects that they are doing well. Notably, no explicit strategies or instructions were given as, with few exceptions(246), implicit strategies have been shown to produce better outcomes(247-252).

Continuous, real-time auditory feedback will be used for reinforcement and produced within 30 milliseconds of the subject's ISFs (0.0-0.1 Hz) within the pre-defined region(s) of interest (i.e. MCC and/or PCC) surpassing the threshold(s). These ROI were selected because, as outlined in the introduction, they are considered key cortical nodes within the core ICNs which are consistently found to be disrupted in ID populations. SLORETA permits the selection of any cortical region for feedback of the current density using voxels selected based on Montreal Neurological Institute (MNI)-coordinates(253). For a complete list of targeted voxels for this trial, see Additional files 1 and 2.

The reward threshold(s) will be manually adjusted in real-time to maintain a $60 \% \pm 10 \%$ success rate. Manual, rather than automated, thresholding was chosen as it has been reported to lead to better EEGlearning $(243,248,250,254)$. The yoked-sham sessions will be identical to active sessions, including live EEG recordings and real-time motion/EMG artefact alerts, however the auditory rewards will derive from playbacks of a series of 6 consecutive ISF-NFB sessions from another female with IDs recorded via free, open-source Audacity software(255) which uses the computer's sound card as an audio to digital converter. Importantly, it has been reported that training effects are more robust when the clinician is present(256) therefore, irrespective of group assignment, the trainer was present for the duration of all sessions. Further, the trainer will monitor subject protocol adherence.

\section{Criteria for discontinuing or modifying allocated interventions $\{11 \mathrm{~b}\}$}

Participants may withdraw at any time without giving a reason or may be withdrawn by the investigators if they are unable to adhere to protocol (i.e. miss $>1$ elSF-NFB session), start or alter other therapies (e.g. start/change medications), or experience significant adverse effects. 


\section{Strategies to improve adherence to interventions $\{11 \mathrm{c}\}$}

We will attempt to mitigate adherence issues via automated email and text message reminders sent on the day of each training session.

\section{Relevant concomitant care permitted or prohibited during the trial $\{11 \mathrm{~d}\}$}

Participants should continue to take medications for other conditions as normal and will be asked to maintain any current first-line mental health therapies (e.g. pharmacotherapy) for the entire length of the study period. Any changes to these first-line therapies (e.g. altered pharmacotherapy dosages, introduction of intensive psychotherapy) will render participants ineligible.

See Table 1 for a detailed description of the trial intervention using the Template for Intervention Description and Replication (TIDieR, (257))

\section{Provisions for post-trial care $\{30\}$}

In the unlikely event of injury, participants will be eligible to apply for compensation from the Accident Compensation Corporation (ACC) of New Zealand just as they would be if they were injured in an accident at work or at home. Although there are private providers abroad (e.g. Asia, North America, Europe), should this trial provide evidence of efficacy, there is currently no access to this therapy within New Zealand.

\section{Outcomes $\{12\}$}

The central importance of PROs in clinical trials has been emphasized by both international health regulatory agencies and patients $(258,259)$, therefore, the primary outcomes of interest are transdiagnostic PROs encompassing the Inventory of Depression and Anxiety Symptoms - Second Version (IDAS-II)(260, 261), Multidimensional Emotional Disorder Inventory (MEDI)(262), and Hospital Anxiety and Depression Scale (HADS)(263). To reduce the risk of type-1 errors (i.e. false positives) due to multiple comparisons, these three PRO measures will be assessed as follows:
a. HADS: total (anxiety + depression) scores
b. IDAS-II: composite (general depression + social anxiety) scores
c. MEDI: composite (neurotic temperament + positive temperament (reverse scored) + depression + social concerns) scores

The aforementioned IDAS-II and MEDI symptom-domains were selected based upon their relatively strong associations with the IDs of interest in our trial (i.e. MDD, GAD, SOC)(260-262).

The secondary outcomes include the Intolerance of Uncertainty Scale (IUS-12)(264), Repetitive Thinking Questionnaire (RTQ-10)(265-267), activity and connectivity within and between core ICNs, heart rate variability (HRV), and electrodermal activity (EDA). 
Outcome measures will be assessed at baseline on two separate occasions approximately 1-week apart followed by mid-training (i.e. between the $6^{\text {th }}$ and $7^{\text {th }}$ ISF-NFB session), 1-week post-training, and 1-month post-training assessments. The duplicate baseline assessments allow us to examine state-variability and test-retest reliability. The mid-training assessments permit comparisons of active vs sham (i.e. active PCC vs sham PCC and active PCC/MCC vs sham PCC/MCC) and single- vs multi-region (i.e. active PCC vs active PCC/MCC) groups. The post 1-week and post 1-month follow-ups allow for the determination of any immediate and delayed treatment effects, respectively, following either 6 or 12 active sessions. For all outcomes, the analysis metric of interest will be group mean (or median) change from baseline.

\section{Participant timeline $\{13\}$}

The trial consists of a 1-week pre-intervention (i.e. baseline assessments) phase, 4-week intervention (i.e. sLORETA eISF-NFB) phase, and 4-week post-intervention (i.e. follow-up) phase[2]. The total trial period will be approximately 9-weeks (see Figure 1 ).

\section{Sample Size $\{14\}$}

Our target sample sizes for our clinical and healthy populations are $n=60$ and $n=60$, respectively, which is based on trial feasibility during the time of recruitment. Based on our prior feasibility study(194), the projected rate of loss to follow-up/discontinuation following randomisation is $10-15 \%$. To date, this is the first study examining the effect of SLORETA eISF-NFB on IDs, thus no formal power calculations can be made. Our research group's previous sLORETA eISF-NFB feasibility study found significant differences in PROs and neurophysiological measures between the active and sham groups with an $n=11$ and $n=10$, respectively $(194,195)$.

\section{Recruitment $\{15\}$}

A convenience sample will be recruited will be via posters and Facebook ads with an invitation to participate in a University of Otago mental health study. Advertisements will direct potential participants to a webpage that will describe the trial and invite those interested to complete an online screening form which will query basic information including first name, age, date of birth, sex, ethnicity, education level, handedness, current diagnoses and medications, pregnancy status, presence of electronic implants (i.e. pacemakers), email address, and phone number. Individuals who pass the preliminary screening will be contacted via email and asked to attend an in-person mental health interview at the University of Otago Hospital, Dunedin, New Zealand. Those that agree will be provided directions to the lab and a digital copy of the 6-7 page participant information sheet. A reminder text will be sent to potential participants on the day of their interview. Each person will be screened using the Mini-International Neuropsychiatric Interview (MINI; English version 7.0.2 for DSM-5)(268) by a extensively trained doctoral student. The MINI is a brief structured diagnostic interview, shown to be both valid and reliable, used to assess the 17 most common psychiatric disorders including MDD, suicidality, bipolar, panic disorder, agoraphobia, SOC, obsessive compulsive disorder, post-traumatic stress disorder, alcohol use disorder, substance use disorder, psychoses, anorexia, bulimia, binge-eating disorder, GAD, and anti-social personality 
disorder $(269,270)$. In the event that the interviewer suspects that the interviewee is at high-risk for suicide, he will screen using the Columbia-Suicide Severity Rating Scale (C-SSRS - Screen Version; (271)) with affirmative answers to questions 4, 5, and/or $6 \mathrm{~b}$ initiating immediate referral to Emergency Psychiatric Services. Ultimately, those meet the inclusion criteria and none of the exclusion criteria will be enrolled into the study, have anthropometric (i.e. height and weight) measurements taken, and scheduled for their baseline assessments. Participants will also be familiarized with the study equipment, procedures, and personnel. Recruitment will continue until our target sample sizes ( $n=60$ clinical and $\mathrm{n}=60$ healthy) is met and is expected to take up to 12 months. All participants who complete the study will receive a $\$ 40$ supermarket voucher as reimbursement for any parking/travel expenses.

\section{Assignment of interventions: allocation}

\section{Sequence generation $\{16 \mathrm{a}\}$}

Randomisation will be performed using the website randomization.com. This tool is a valid randomisation program utilised by clinical trial researchers. To ensure balanced sample size across the groups over time, block randomisation with random block sizes and a 1:1:1:1 allocation will be utilised.

\section{Concealment mechanism $\{16 \mathrm{~b}\}$}

To ensure concealment, the block sizes will not be disclosed. Further, treatment conditions will be kept in sequentially number, sealed, opaque envelopes in the central office.

\section{Implementation $\{16 c\}$}

A lab member from the group who has no direct contact with the participants will conduct the randomisation process. TMP is responsible for participant enrolment and will assign participants to interventions following baseline assessments and confirmation of scheduled elSF-NFB sessions.

\section{Assignment of interventions: Blinding}

\section{Who will be blinded $\{17 a\}$}

This is a double-blind study whereby trial subjects and outcome assessors will be unaware of group assignments. In an effort to improve blinding, all aspects of sham sessions will be identical to active sessions including the live recording of sham participants' EEGs along with real-time artefact alerts. Blinding integrity will be assessed during the mid-training assessment session via an IPad whereby participants will be queried as to 1) their perceived group allocation, 2) confidence in their answer to question 1 on a scale of $0-100 \%, 3$ ) reason for their answer to question 1, and 4) if their group assignment was revealed to them in any way.

\section{Procedure for unblinding if needed $\{17 b\}$}

Treatment assignment will be disclosed to trial participants only upon their completion of the study. 


\section{Data collection and management}

\section{Plans for assessment and collection of outcomes $\{18 \mathrm{a}\}$}

All assessment sessions for a given subject will take place at approximately the same time of day and be led by a female research assistant. Prior to each assessment session, participants will be asked to abstain from 1) food and water for 2 hours, 2) smoking/vaping for 8 hours, 3) strenuous exercise, alcohol, caffeine \& over-the-counter medication for 24 hours. A reminder email and text will be sent to each participant one day prior to and on the day of assessment sessions, respectively, in an effort to increase adherence. Adherence to lifestyle restrictions will be queried at the beginning of each session with any breaches recorded. In addition, the subject's previous night's sleep duration will be documented and they will be asked to use the toilet immediately prior to testing to ensure that their bladders are empty. Together, these standardisation procedures will help to control for state-variability stemming from influences like circadian rhythms(272), satiety(273), sleep duration(55), hydration levels(274), and smoking(275) and are in line with current recommendations for neurophysiological data collection(276). Further, two baseline assessments will be acquired on each participant approximately 1 week apart in order to assess state-variability and test-retest reliability. Duplicate baseline follows guidance to improve the reliability of brain imaging-related metrics via multiple (i.e. >1) resting-state scans(277-280).

All PROs are English versions and have been created in digital form via Qualtrics(281) which will allow participants to complete them using an IPad during their EEG set-up and prior to all neurophysiological assessments. The order of PRO administration will be standardized and based on PRO length (i.e. IDAS-II $>$ MEDI > HADS > IUS-12 > RTQ-10). To prevent missing data, a visual alert will be generated if any queries on a given form have missing responses. Research has indicated the electronic data collection increases the speed, accuracy, and user acceptability of the process(282-284). The estimated total time to complete the battery of PROs is 20 minutes.

\section{Primary outcomes}

The HADS is a valid and reliable 14-item, trans-diagnostic PRO measure used to assess anxiety and depression severities(263). Response options are on a 4-point scale (0-3) based on participants experiences over the past week with anxiety and depression subscale scores graded as follows: 0$7=$ normal, 8-10=borderline abnormal/borderline case, 11-21=abnormal/case(263, 285). The HADS has been repeatedly shown to be a reliable and valid tool across a variety of settings(285-287). There is some debate with respect to whether the HADS is best assessed via the total 14-item score(288-290) or two 7-item subscale (anxiety and depression) scores $(285,287,290,291)$.

The IDAS-II is a valid and reliable 99-item, trans-diagnostic PRO measure that uses a response scale ranging from 1 (not at all) to 5 (extremely) to assess 19 current (past 2 weeks) ID-related symptom domains including general depression (20-items), dysphoria (10-items), lassitude (6-items), insomnia (6items), suicidality (6-items), appetite loss (3-items), appetite gain (3-items), well-being (8-items), ill temper (5-items), mania (5-items), euphoria (5-items), panic (8-items), social anxiety (6-items), claustrophobia (5- 
items), traumatic intrusions (4-items), traumatic avoidance (4-items), checking (3-items), ordering (5items), and cleaning (7-items) $(260,261)$. Notably, in contrast to the other domains, the general depression domain is a composite of all 10 items from the dysphoria domain, as well as 2 items each from the suicidality, lassitude, insomnia, appetite loss and well-being domains. Recently, severity (mild, moderate, severe) thresholds have been introduced for 12 of the subscales including general depression, dysphoria, lassitude, insomnia, suicidality, appetite loss, appetite gain, well-being, ill-temper, panic, social anxiety, and traumatic intrusions(292).

The MEDI is a valid and reliable 49-item, trans-diagnostic PRO measure that employs a response scale ranging from 0 (not characteristic of me/does not apply to me) to 8 (extremely characteristic of me/applies to me very much) to assess nine ID-related symptom domains, originally proposed by Brown and Barlow(293) which include 1) Neurotic Temperament, 2) Positive Temperament, 3) Depression, 4) Autonomic Arousal, 5) Somatic Anxiety, 6) Intrusive Cognition, 7) Social Concerns, 8) Traumatic Reexperiencing, and 9) Avoidance $(262,294)$. Although validated clinical severity thresholds for the MEDI total subscale scores are still lacking, the authors have suggested that average subscale scores $>4$ (or $<4$ for positive temperament), $>6$ (or $<2$ for positive temperament), and 7-8 (or 0-1 for positive temperament) may reflect moderate, severe, and extreme severities, respectively(295).

\section{Secondary Outcomes}

The IUS-12 is a valid and reliable 12-item, transdiagnostic PRO measure that assesses the degree to which an individual considers the possibility of a negative event occurring unacceptable, irrespective of its probability of occurrence $(264,296)$. Whereas the original IUS- 27 was GAD-specific, the IUS- 12 has been distilled in order to measure the core intolerance of uncertainty construct(297). The IUS-12 uses a response scale from 1 (not at all characteristic of me) to 5 (entirely characteristic of me)(264). Notably, although some researchers have claimed that the IUS-12 is a unidimensional construct and recommend using only the IUS-12 total score(298-300), there has been considerable support for a two-factor IUS-12 structure: 1) a 7-item prospective IU scale related to action/approach-oriented strategies in order to increase certainty (e.g. seeking more information), and 2) a 5-item inhibitory IU associated with inaction/avoidance-oriented thoughts and behaviours (e.g. delayed decision making)(264, 296, 301-308). Notably, IUS is a common trait shared across the ID spectrum(308-311).

The RTQ-10 a 10-item, trans-diagnostic PRO measure(266) distilled from three disorder-specific scales: 1) the MDD-associated Ruminative Responses Scale (RRS; (312)), 2) the GAD-associated Penn State Worry Questionnaire (PSWQ; (313)), and 3) the SOC-associated Post-Event Processing Questionnaire-Revised (PEPQ-R; (314)). Importantly, repetitive negative thinking (i.e. rumination and worry) is a characteristic feature of IDs $(74,315,316)$.

Resting-state full-band EEGs (fb-EEGs), electrocardiograms (ECGs), and EDAs will be collected concurrently with a Compumedics Neuroscan SynAmps RT DC/AC amplifier using a continuous sampling rate of $1000 \mathrm{~Hz}$ and $50 \mathrm{~Hz}$ notch filter. Recordings will take place in a quiet, cool $\left(\sim 15^{\circ} \mathrm{C}\right)$, dimly lit room 
as participants are seated upright in a comfortable chair with their eyes closed. Notably, EEG reliability appears to be improved in the eyes closed condition $(317,318)$.

10- minute resting-state fb-EEGs will use high-density (64-channel) silicone Quik-Cap Hydro Net caps with $\mathrm{Ag} / \mathrm{AgCl}$ electrode placements corresponding to the international extended $10 / 20$ system. The ground electrode is positioned at $\mathrm{AFz}$ with the reference electrode midway between $\mathrm{Cz}$ and $\mathrm{CPz}$.

Electrooculography (EOG) will track vertical and horizontal eye movement artefacts. The cap is soaked in a saline solution at least 30 minutes prior to application and all electrode impedances will be kept below $10 \mathrm{k} \Omega$. To help reduce impedances, subjects were asked to arrive with non-braided, dry, clean (i.e. no conditioner, gels, pastes, sprays) hair. fb-EEG recordings will be for 10-minutes.

10-minute resting-state EDA (in microsiemens, $\mu \mathrm{S}$ ) will assessed with bipolar finger $\mathrm{Ag} / \mathrm{AgCl}$ electrodes securely attached to the palmar surface of the middle phalanxes of the $4^{\text {th }}$ and $5^{\text {th }}$ digits (i.e. ring and little fingers) of the non-dominant hand.

Resting-state heart rate will be monitored using a standard limb lead (lead-II) ECG at rest using $\mathrm{Ag} / \mathrm{AgCl}$ electrodes. A 10-minute spontaneous breathing ECG will be collected concurrently with the other neurophysiological assessments followed immediately by a 10-minute metronome paced breathing (12 breaths per minute) ECG with a 1:1 inspiratory/expiratory (I/E) ratio (i.e. $2.5 \mathrm{sec}$ inhalation/2.5 sec exhalation). Participants will be instructed to breathe through their nose and at normal depth (i.e. no deep breathing).

\section{Plans to promote participant retention and complete follow-up $\{18 \mathrm{~b}\}$}

Once enrolled, every reasonable effort will be made to follow participants throughout the entirety of the study period via ongoing email and text messaging correspondence. In the event of premature discontinuation of the study for any reason, participants will be made aware that all data collected up to the point of withdrawal may be used for analyses.

\section{Data management $\{19\}$}

Participant paper files, including case-report-forms and MINI assessments, are to be kept in numerical order and stored in a locked file drawer in the lead researcher's office. PROs will be electronically stored in Qualtrics with a back-up copy automatically generated and sent to the lead researcher's trial email address. All data collected will be entered into Microsoft Excel (2016) and double-checked for accuracy by the data analyst at the time of entry. Participant data will be maintained for a period of not less than 10 years after the completion of the study.

\section{Confidentiality $\{27\}$}

All information generated in this study will be considered highly confidential and is not to be shared with any persons not directly concerned with the study. For de-identification purposes, participants will be assigned unique study numbers upon enrolment (e.g. ISAD_F8 $=8^{\text {th }}$ enrolled female in the ISAD trial). All 
electronic records will be identified solely using assigned study numbers and stored locally in a password protected database. All paper records will be stored on-site in a locked office accessible only to the researchers directly involved in the trial. Further, paper documents that contain personal identifiers (i.e. informed consent forms), will be store separately from de-identified paper records (i.e. case-report-forms and MINI assessments).

\section{Plans for collection, laboratory evaluation and storage of biological specimens for genetic or molecular analysis in this trial/future use $\{33\}$ an}

Not applicable: no specimens collected

\section{Statistical methods}

\section{Statistical methods for primary and secondary outcomes $\{20 \mathrm{a}\}$}

SPSS (version 27) will be used for PRO statistical analyses. PRO group means/standard deviations will be calculated at each time point. Baseline \#1 and \#2 values will be used to assess test-retest reliability for all PROs in both the non-ID and ID cohorts. An independent t-test (95\% confidence intervals) will be used to compare baseline \#2 values of the non-ID and ID cohorts.

In our ID cohort, change from baseline \#2 values will be statistically compared using repeated measures ANOVA (95\% confidence intervals) with group allocation as the between-subjects factor and time as a within-subjects factor. Change from baseline will be considered at three time-points: 1) mid-training (i.e. between session 6 and 7), 2) 1-week post-training, and 3) 1-month post-training. Due to the large number of comparison, appropriate controls for multiple comparisons will be applied. In addition, if feasible, we will undertake Bayesian analyses in $\mathrm{R}$ (version 4.0.2; (319)) with posterior distributions and corresponding $95 \%$ credibility intervals reported.

For fb-EEGs, raw data will be resampled at $128 \mathrm{~Hz}$, filtered from $0.001 \mathrm{~Hz}$ to $44 \mathrm{~Hz}$, plotted, and carefully inspected using manual and/or automated artefact rejection in EEGLAB (version 2020). Subsequently, average cross-spectral matrices will be computed for bands infraslow $(0.01-0.1 \mathrm{~Hz})$, slow $(0.1-1 \mathrm{~Hz})$, delta (1-4 Hz), theta (4-8 Hz), alpha (8-12 Hz), beta $(12-30 \mathrm{~Hz})$ and gamma $(30-44 \mathrm{~Hz})$. A validated sourcelocalization technique, exact LORETA (eLORETA) $(320,321)$, will be used to in order to determine intracerebral electrical sources of the scalp-recorded EEG activity in each of the frequency bands. Using the Montreal Neurological Institute (MNI)-152 template, eLORETA produces an inverse solution space consisting of 6239 cortical grey matter voxels at $5 \mathrm{~mm}$ resolution and has been shown to produce exact, zero-error localisations even in the presence of structured noise(321). The eLORETA software package performs the statistical analyses using a non-parametric methodology. It is based on estimating, via randomization, the empirical probability distribution for the max-statistic (e.g. the maximum of a $t$ or an $F$ statistic), under the null hypothesis while correcting for multiple testing (i.e., for the collection of tests performed for all electrodes and/or voxels, and for all time samples and/or discrete frequencies). The interested reader is referred to (322) for a complete overview of the methodology, where details about the 
properties (e.g. pertaining to its non-parametric nature, and pertaining to how it properly corrects for multiple testing) can be found

The raw ECG signal will be band-passed, differentiated, squared, integrated, and smoothed to isolate Rwaves. Manual inspection of the ECG trace will be performed for artefact (e.g. ectopic beats) identification and correction. Short-term (5-10 min) HRV using both spontaneous- and paced-breathing ECGs will be calculated using standard (low $=0.04-0.15 \mathrm{~Hz}$ and high $=0.15-0.4 \mathrm{~Hz}$ ) frequency- and timedomain analyses with either HRVTool (version 1.07; (323)) or RHRV (version 4.2.6; (324)) software.

Skin conductance levels (SCLs) and non-specific skin conductance responses (ns-SCRs; threshold 0.05 $\mu S)$ will be tracked and analysed via Breathe Easy EDA (BEEDA; (325) or PsychoPhysiological Modelling (PsPM; version 4.2.1; (326)) software.

\section{Interim analyses $\{21 \mathrm{~b}\}$}

Not applicable: no interim analyses will be performed and no stopping guidelines will be established.

\section{Methods for additional analyses (e.g. subgroup analyses) \{20b\}}

If primary outcomes do not significantly differ between active groups at each time point, combined between-group comparisons (active PCC \& PCC/MCC vs sham>active PCC \& PCC/MCC) will also be performed using the methods outlined above.

\section{Methods in analysis to handle protocol non-adherence and any statistical methods to handle missing data $\{20 \mathrm{c}\}$}

For data to be included in the current study, participants had to schedule and complete a minimum of 10 out of 12 elSF-NFB sessions. Modern imputation methods will be applied for missing data reflecting uncertainty in the modelling process and inherent variability in patient outcomes. Further, we will report and qualitatively compare reasons for withdrawal in each of the randomisation groups. Based on our prior feasibility study(194), discontinuation/loss-to-follow-up during the intervention/post-intervention phases is expected to be $10-15 \%$.

\section{Plans to give access to the full protocol, participant level-data and statistical code $\{31 \mathrm{c}\}$}

The full protocol will be published in a peer-reviewed, open-source journal prior to recruitment completion. No more than 2 years following the final data collection, we will deliver the completed, de-identified dataset and statistical code to the appropriate data archive for sharing purposes in line with the scientific imperatives of increased transparency, reproducibility, and interpretation of trials.

\section{Oversight and monitoring}

Composition of the coordinating centre and trial steering committee $\{5 \mathrm{~d}\}$ 
Not applicable: no coordinating centre or trial steering committee for this trial

\section{Composition of the data monitoring committee, its role and reporting structure $\{21 \mathrm{a}\}$}

Due to the relatively short duration of recruitment, non-invasive make-up of the procedures/interventions, and non-serious nature of adverse effects reported in our prior feasibility trial, no formal data monitoring committee will be established.

\section{Adverse event reporting and harms $\{22\}$}

We will systematically monitor adverse effects from the therapy for the duration of the trial using the Discontinuation-Emergent Signs and Symptoms checklist (DESS; (327)) created, verbatim, in Qualtrics and completed by participants on an iPad during EEG set-ups in the interventional and post-interventional phases. Initially developed for drug trials(327), the DESS is a structured 43-item self-report that utilises the following scale: $1=$ new symptom, $2=$ old symptom but worse, $3=$ old symptom but improved, $4=$ old symptom but unchanged, $5=$ symptom not present. The DESS has been used for the assessment of treatment-related side-effects in ID populations $(328,329)$ and, recently, has been employed to monitor adverse-effects specifically associated with NFB therapy(330). Participants may be withdrawn from the trial by the investigators, even without their request, in the event of serious adverse effects. As detailed in the PIS, a brief (4-item) interview used during our group's prior sLORETA eISF-NFB feasibility trial revealed that, although unusual or vivid dreams were experienced by some participants, there were no serious adverse effects(195).

\section{Frequency and plans for auditing trial conduct $\{23\}$}

Not applicable: no auditing of trial conduct will be performed.

\section{Plans for communicating important protocol amendments to relevant parties (e.g. trial participants, ethical committees) $\{25\}$}

Substantive protocol amendments which may impact on the conduct of the study including changes to the study objectives, design, population, sample sizes, or procedures will be agreed upon by the research team, updated in the trial registry, submitted to the ethics committee for approval and updated on our online trial advertisements and web pages.

\section{Dissemination plans $\{31$ a $\}$}

Every effort will be made to minimise the interval between the completion of data collection and release of study results. We estimate this process to take 6-12 months. Irrespective of magnitude or direction of effect, results from the study will be written up and submitted to international peer-reviewed scientific journals, presented at scientific conferences, and may form part of grant applications. In addition, once compiled, all participants will be provided with a digital copy of the results.

[1] The criterion does not apply to non-ID controls. 
[2] The non-ID group will undergo the MINI and baseline assessments only.

\section{Discussion}

Approximately one in five New Zealander's is dealing with a mental illness at any given time with the majority experiencing psychopathology at some point in their lifetime(7). Alarmingly, New Zealand's suicide numbers are increasing with the 2017-18 rate the highest it's been in 20 years(7).

Psychopathology has been estimated to reduce life expectancy by up to 25 years(7). A recent government inquiry by the New Zealand government has shed light on the shortcomings of current treatment and called for wider implementation of non-pharmaceutical approaches in treatment of mental health problems(7). Similarly, scientists in other parts of the world are calling for research into 'novel interventions that may be based on altering plasticity or returning circuitry rather than neurotransmitter pharmacology'(331).

The implementation of safe, non-invasive neuromodulation techniques that have the potential to impact neuroplasticity within and between large-scale ICNs may offer new treatment opportunities for individuals who either do not want, respond to, or tolerate standard interventions. Additionally, these techniques may serve as adjuncts to traditional treatments, potentially enhancing their efficacy. To date, ours is the only research group studying the effects of this novel therapy in clinical populations. We believe targeting core ICNs via sLORETA eISF-NFB holds promise in the treatment of psychopathologies like IDs.

\section{Trial Status}

Version 1: 15 October, 2019.

Version 2: 15 May, 2020.

Recruitment began on 15 February, 2020 but was prematurely halted due to COVID-19 lockdown measures here in New Zealand. Recruitment efforts resumed on 15 June, 2020, however, due to budgetary and time restrictions imposed by the aforementioned measures, we amended our protocol. Specifically, our recruitment goal for clinical participants was changed from 80 (40 males and 40 females) to 60 females. We anticipate recruitment of our clinical and 'healthy' cohorts to be completed sometime between 15 Jun-15 Dec, 2021.

\section{Abbreviations}

ADHD = Attention deficit hyperactivity disorder

alNS = anterior Insula

ANOVA $=$ Analysis of variance 
ANZCTR = Australia New Zealand Clinical Trials Registry

CEN $=$ Central executive network

COVID-19 = Coronavirus Disease 2019

DESS = Discontinuation-emergent signs and symptoms checklist

dIPFC $=$ dorso-lateral Prefrontal cortex

DMN = Default mode network

DSM-5 $=$ Diagnostic and statistical manual of mental disorders $-5^{\text {th }}$ Edition

ECG $=$ Electrocardiography

EDA $=$ Electrodermal activity

EEG $=$ Electroencephalography

EEG-NFB = Electroencephalography neurofeedback

elSFs = electrophysiological Infraslow fluctuations

elSF-NFB = electrophysiological Infraslow fluctuation neurofeedback

eLORETA = exact low-resolution electromagnetic tomography

$E M G=$ Electromyography

$E O G=$ Electrooculography

$\mathrm{GAD}=$ Generalised anxiety disorder

HADS = Hospital Anxiety and Depression Scale

$\mathrm{HRV}=$ Heart rate variability

$\mathrm{ICN}=$ Intrinsic connectivity network

IDs $=$ Internalising Disorders

IDAS-II = Inventory of Depression and Anxiety Symptoms - Second Version

ISAD trial = Infraslow neurofeedback for Anxiety and Depression trial

IUS-12 = Intolerance of Uncertainty Scale - 12-item short form 
MCC $=$ Mid-cingulate cortex

MDD = Major depressive disorder

MEDI = Multidimensional Emotional Disorder Inventory

MINI = Mini-International Neuropsychiatric Interview

MNI = Montreal Neurological Institute

$\mathrm{mPFC}=$ medial Prefrontal cortex

NFB $=$ Neurofeedback

ns-SCR = non-specific Skin conductance response

PCC $=$ Posterior cingulate cortex

PROs $=$ Patient reported outcomes

RTQ-10 = Repetitive Thinking Questionnaire - 10-item trait version

$\mathrm{SCL}=$ Skin conductance level

SOC $=$ Social Anxiety Disorder

SLORETA = standardized low-resolution electromagnetic tomography

$\mathrm{SN}=$ Salience network

$\mu \mathrm{S}=$ microsiemens

\section{Declarations}

\section{Acknowledgements}

We are grateful to all of the trial participants for their willingness and commitment to participate in this trial.

\section{Authors' contributions $\{31 \mathrm{~b}\}$}

TMP conceived of the study and drafted the protocol. TMP, PG, DDR, and DA contributed to the refinement of the trial protocol. TMP will conduct the trial, statistical analyses and dissemination of results. PG provided expertise on mental health disorders. DDR provided neuroscientific expertise. DA provided statistical expertise. MS created the neurofeedback software programs and provided training in their implementation. All authors read, provided feedback, and approved the final manuscript. 


\section{Funding $\{4\}$}

This clinical trial is part of a doctoral thesis supported by the Department of Surgical Sciences, University of Otago, Dunedin, New Zealand.

\section{Availability of data and materials $\{29\}$}

The final trial dataset will be password protected and housed locally at the research lab. Other team members will be provided access to this dataset by TMP upon request. To ensure confidentiality, data dispersed to project team members will be blinded of any identifying participant information.

\section{Ethics approval and consent to participate $\{24\}$}

Ethical approval has been obtained from the Health \& Disability Ethics Committee (HDEC), New Zealand (19/CEN/179)[see Additional file 3] and The Ngāi Tahu Research Consultation Committee was consulted. Written, informed consent to participate will be obtained from all participants.

\section{Consent for publication $\{32\}$}

Examples of the participant-information and informed-consent forms can be provided upon request.

\section{Competing interests $\{28\}$}

MS is the owner of Neurofeedback Therapy Services of New York which provides ISF-NFB therapy for patients and training for clinicians. He created the ISF-NFB software programs for this trial, provided guidance to the principal investigator in their utilisation, and participated in editing of the final version of the manuscript. MS did not participate in the trial conceptualisation, design, implementation, or analyses. All other authors declare no competing interests.

\section{References}

1. Kessler RC, Aguilar-Gaxiola S, Alonso J, Chatterji S, Lee S, Ormel J, et al. The global burden of mental disorders: an update from the WHO World Mental Health (WMH) surveys. Epidemiologia e psichiatria sociale. 2009;18(1):23-33.

2. Pfeifer JH, Allen NB. Puberty initiates cascading relationships between neurodevelopmental, social, and internalizing processes across adolescence. Biological Psychiatry. 2020.

3. Haidt J, Allen N. Scrutinizing the effects of digital technology on mental health. Nature Publishing Group; 2020.

4. Keyes KM, Dahsan G, Patrick MOM, Hamilton A, Schulenberg J. Recent increases in depressive symptoms among US adolescents: trends from 1991 to 2018. Social Psychiatry and Psychiatric Epidemiology. 2019;54(8):987-96. 
5. Duffy ME, Twenge JM, Joiner TE. Trends in Mood and Anxiety Symptoms and Suicide-Related Outcomes Among U.S. Undergraduates, 2007-2018: Evidence From Two National Surveys. Journal of Adolescent Health. 2019;65(5):590-8.

6. Twenge JM, Cooper AB, Joiner TE, Duffy ME, Binau SG. Age, period, and cohort trends in mood disorder indicators and suicide-related outcomes in a nationally representative dataset, 2005-2017. Journal of abnormal psychology. 2019;128(3):185.

7. Kris N. 'Once in a generation': The crucial passages from the mental health inquiry. 2018.

8. Buchan $\mathrm{H}$, Sunderland $\mathrm{M}$, Carragher $\mathrm{N}$, Batterham $\mathrm{P}$, Slade T. Investigating age-related differences in responses to screening items for internalising disorders in three national surveys. Journal of Affective Disorders. 2014;152-154:229-36.

9. Cosgrove VE, Rhee SH, Gelhorn HL, Boeldt D, Corley RC, Ehringer MA, et al. Structure and etiology of co-occurring internalizing and externalizing disorders in adolescents. Journal of abnormal child psychology. 2011;39(1):109-23.

10. Altemus M, Sarvaiya N, Neill Epperson C. Sex differences in anxiety and depression clinical perspectives. Frontiers in neuroendocrinology. 2014;35(3):320-30.

11. Asher M, Asnaani A, Aderka IM. Gender differences in social anxiety disorder: A review. Clinical Psychology Review. 2017;56:1-12.

12. Bandelow B, Michaelis S. Epidemiology of anxiety disorders in the 21 st century. Dialogues in clinical neuroscience. 2015;17(3):327.

13. Hammen C. Risk Factors for Depression: An Autobiographical Review. Annual Review of Clinical Psychology. 2018;14(1):1-28.

14. Hoertel N, McMahon K, Olfson M, Wall MM, Rodríguez-Fernández JM, Lemogne $C$, et al. A dimensional liability model of age differences in mental disorder prevalence: Evidence from a national sample. Journal of Psychiatric Research. 2015;64:107-13.

15. de Graaf R, ten Have M, van Gool C, van Dorsselaer S. Prevalence of mental disorders and trends from 1996 to 2009. Results from the Netherlands Mental Health Survey and Incidence Study-2. Social Psychiatry and Psychiatric Epidemiology. 2012;47(2):203-13.

16. Blanco C, Vesga-López O, Stewart JW, Liu S-M, Grant BF, Hasin DS. Epidemiology of major depression with atypical features: results from the National Epidemiologic Survey on Alcohol and Related Conditions (NESARC). The Journal of clinical psychiatry. 2012;73(2):224.

17. Ohayon MM, Schatzberg AF. Social phobia and depression: Prevalence and comorbidity. Journal of Psychosomatic Research. 2010;68(3):235-43.

18. Stein DJ, Lim CCW, Roest AM, de Jonge P, Aguilar-Gaxiola S, Al-Hamzawi A, et al. The cross-national epidemiology of social anxiety disorder: Data from the World Mental Health Survey Initiative. BMC medicine. 2017;15(1).

19. de Graaf R, ten Have M, Tuithof M, van Dorsselaer S. First-incidence of DSM-IV mood, anxiety and substance use disorders and its determinants: Results from the Netherlands Mental Health Survey and Incidence Study-2. Journal of Affective Disorders. 2013;149(1):100-7. 
20. McDowell RD, Ryan A, Bunting BP, O'Neill SM, Alonso J, Bruffaerts R, et al. Mood and anxiety disorders across the adult lifespan: a European perspective. 2014;44(4):707-22.

21. Kessler RC, Sampson NA, Berglund P, Gruber MJ, Al-Hamzawi A, Andrade L, et al. Anxious and nonanxious major depressive disorder in the World Health Organization World Mental Health Surveys. Epidemiology and psychiatric sciences. 2015;24(3):210.

22. Kessler RC, Bromet EJ. The Epidemiology of Depression Across Cultures. Annual Review of Public Health. 2013;34(1):119-38.

23. Oakley-Browne M, Wells JE, Scott KM, New Zealand. Ministry of $H$. Te rau hinengaro the New Zealand mental health survey. Wellington, N.Z.: Ministry of Health; 2006.

24. Kessler RC, Angermeyer M, Anthony JC, De Graaf R, Demyttenaere K, Gasquet I, et al. Lifetime prevalence and age-of-onset distributions of mental disorders in the World Health Organization's World Mental Health Survey Initiative. World psychiatry : official journal of the World Psychiatric Association (WPA). 2007;6(3):168.

25. Perlman K, Benrimoh D, Israel S, Rollins C, Brown E, Tunteng J-F, et al. A systematic meta-review of predictors of antidepressant treatment outcome in major depressive disorder. Journal of affective disorders. 2019;243:503-15.

26. Fornaro M, Anastasia A, Novello S, Fusco A, Pariano R, De Berardis D, et al. The emergence of loss of efficacy during antidepressant drug treatment for major depressive disorder: an integrative review of evidence, mechanisms, and clinical implications. Pharmacological research. 2019;139:494-502.

27. losifescu D. Electroencephalography-Derived Biomarkers of Antidepressant Response. Harvard Review of Psychiatry. 2011;19(3):144-54.

28. Furukawa TA, Kato T, Shinagawa $Y$, Miki K, Fujita $H$, Tsujino $N$, et al. Prediction of remission in pharmacotherapy of untreated major depression: development and validation of multivariable prediction models. Psychological medicine. 2019;49(14):2405-13.

29. Choi KW, Jeon HJ. Heart Rate Variability for the Prediction of Treatment Response in Major Depressive Disorder. Frontiers in Psychiatry. 2020;11:607.

30. Nicholson AA, Ros T, Jetly R, Lanius RA. Regulating posttraumatic stress disorder symptoms with neurofeedback: Regaining control of the mind. Journal of Military, Veteran and Family Health. 2020;6(S1):3-15.

31. Seibell PJ, Hollander E. Management of obsessive-compulsive disorder. F1000prime reports. 2014;6:68-.

32. Haller SPW, Cohen Kadosh K, Scerif G, Lau JYF. Social anxiety disorder in adolescence: How developmental cognitive neuroscience findings may shape understanding and interventions for psychopathology. Developmental Cognitive Neuroscience. 2015;13(C):11-20.

33. Möller H-J, Bandelow B, Volz H-P, Barnikol UB, Seifritz E, Kasper S. The relevance of 'mixed anxiety and depression' as a diagnostic category in clinical practice. European Archives of Psychiatry and Clinical Neuroscience. 2016;266(8):725-36. 
34. Andrade LH, Alonso J, Mneimneh Z, Wells JE, Al-Hamzawi A, Borges G, et al. Barriers to mental health treatment: results from the WHO World Mental Health surveys. 2014;44(6):1303-17.

35. Schoenberg P, David A. Biofeedback for Psychiatric Disorders: A Systematic Review. Applied Psychophysiology and Biofeedback. 2014;39(2):109-35.

36. Pinter A, Szatmari S, Jr., Horvath T, Penzlin Al, Barlinn K, Siepmann M, et al. Cardiac dysautonomia in depression - heart rate variability biofeedback as a potential add-on therapy. Neuropsychiatric disease and treatment. 2019;15:1287-310.

37. Gøtzsche PC. Why I think antidepressants cause more harm than good. The lancet Psychiatry. 2014;1(2):104.

38. Andersohn F, Schade R, Suissa S, Garbe E. Long-term use of antidepressants for depressive disorders and the risk of diabetes mellitus. The American journal of psychiatry. 2009;166(5):591-8.

39. Alvares GA, Quintana DS, Hickie IB, Guastella AJ. Autonomic nervous system dysfunction in psychiatric disorders and the impact of psychotropic medications: a systematic review and metaanalysis. Journal of psychiatry \& neuroscience : JPN. 2016;41(2):89-104.

40. Kim Y-K, Yoon H-K. Common and distinct brain networks underlying panic and social anxiety disorders. Progress in Neuropsychopharmacology \& Biological Psychiatry. 2018;80(Pt B):115-22.

41. Menon V. Large-scale brain networks and psychopathology: a unifying triple network model. Trends in Cognitive Sciences. 2011;15(10):483-506.

42. Xu J, Van Dam NT, Feng C, Luo Y, Ai H, Gu R, et al. Anxious brain networks: A coordinate-based activation likelihood estimation meta-analysis of resting-state functional connectivity studies in anxiety. Neurosci Biobehav Rev. 2019;96:21-30.

43. Hamilton JP, Farmer M, Fogelman P, Gotlib IH. Depressive Rumination, the Default-Mode Network, and the Dark Matter of Clinical Neuroscience. Biological Psychiatry. 2015.

44. Buckner RL, Andrews-Hanna JR, Schacter DL. The Brain's Default Network. Annals of the New York Academy of Sciences. 2008;1124(1):1-38.

45. Raichle M. The Brain's Default Mode Network. Annual Review of Neuroscience. 2015;38:433-.

46. Uddin LQ, Supekar KS, Ryali S, Menon V. Dynamic Reconfiguration of Structural and Functional Connectivity Across Core Neurocognitive Brain Networks with Development. The Journal of Neuroscience. 2011;31(50):18578.

47. Sridharan D, Levitin DJ, Menon V. critical role for the right fronto-insular cortex in switching between central-executive and default-mode networks. P Natl Acad Sci USA. 2008;105(34):12569-74.

48. Elton A, Gao W. Divergent task-dependent functional connectivity of executive control and salience networks. Cortex. 2014;51(1):56-66.

49. Uddin LQ. Salience processing and insular cortical function and dysfunction. Nature Reviews Neuroscience. 2014;16:55.

50. Liang X, Zou Q, He Y, Yang Y. Topologically Reorganized Connectivity Architecture of Default-Mode, Executive-Control, and Salience Networks across Working Memory Task Loads. Cerebral Cortex. 
2016;26(4):1501-11.

51. Dosenbach NUF, Fair DA, Cohen AL, Schlaggar BL, Petersen SE. A dual-networks architecture of topdown control. 2008. p. 99-105.

52. Dai L, Zhou H, Xu X, Zuo Z. Brain structural and functional changes in patients with major depressive disorder: a literature review. PeerJ. 2019;7:e8170.

53. Seeley WW, Menon V, Schatzberg AF, Keller J, Glover GH, Kenna H, et al. Dissociable intrinsic connectivity networks for salience processing and executive control. J Neurosci. 2007;27(9):2349-56.

54. Menon V. Salience Network2015. 597-611 p.

55. Ben Simon E, Rossi A, Harvey AG, Walker MP. Overanxious and underslept. Nature Human Behaviour. 2019.

56. Cersosimo MG, Benarroch EE. Chapter 5 - Central control of autonomic function and involvement in neurodegenerative disorders. In: Buijs RM, Swaab DF, editors. Handbook of Clinical Neurology. 117: Elsevier; 2013. p. 45-57.

57. Critchley HD, Nagai Y, Gray MA, Mathias CJ. Dissecting axes of autonomic control in humans: Insights from neuroimaging. Auton Neurosci-Basic. 2011;161(1-2):34-42.

58. Palma EJ-A, Benarroch EE. Neural control of the heart: Recent concepts and clinical correlations. Neurology. 2014;83(3):261-71.

59. Beissner F, Meissner K, Bar KJ, Napadow V. The Autonomic Brain: An Activation Likelihood Estimation Meta-Analysis for Central Processing of Autonomic Function. J Neurosci. 2013;33(25):10503-+.

60. Sie J-H, Chen Y-H, Shiau Y-H, Chu W-C. Gender- and Age-Specific Differences in Resting-State Functional Connectivity of the Central Autonomic Network in Adulthood. Frontiers in Human Neuroscience. 2019;13.

61. Beauchaine TP. Respiratory Sinus Arrhythmia: A Transdiagnostic Biomarker of Emotion Dysregulation and Psychopathology. Current opinion in psychology. 2015;3:43.

62. Rechlin T, Weis M, Spitzer A, Kaschka WP. Are affective disorders associated with alterations of heart rate variability? Journal of Affective Disorders. 1994;32(4):271-5.

63. Koschke M, Boettger MK, Schulz S, Berger S, Terhaar J, Voss A, et al. Autonomy of autonomic dysfunction in major depression. Psychosomatic Medicine. 2009;71(8):852-60.

64. Ottaviani C, Thayer JF, Verkuil B, Lonigro A, Medea B, Couyoumdjian A, et al. Physiological concomitants of perseverative cognition: A systematic review and meta-analysis. Psychol Bull. 2016;142(3):231-59.

65. Iseger TA, van Bueren NER, Kenemans JL, Gevirtz R, Arns M. A frontal-vagal network theory for Major Depressive Disorder: Implications for optimizing neuromodulation techniques. Brain Stimulation. 2020;13(1):1-9.

66. Koenig J, Kemp AH, Beauchaine TP, Thayer JF, Kaess M. Depression and resting state heart rate variability in children and adolescents-a systematic review and meta-analysis. Clinical psychology 
review. 2016;46:136-50.

67. Nugent AC, Bain EE, Thayer JF, Sollers JJ, Drevets WC. Heart rate variability during motor and cognitive tasks in females with major depressive disorder. Psychiatry Research: Neuroimaging. 2011;191(1):1-8.

68. Liang C-S, Lee J-F, Chen C-C, Chang Y-C. Reactive heart rate variability in male patients with firstepisode major depressive disorder. Progress in Neuropsychopharmacology \& Biological Psychiatry. 2015;56:52-7.

69. Chen X, Yang R, Kuang D, Zhang L, Lv R, Huang X, et al. Heart rate variability in patients with major depression disorder during a clinical autonomic test. Psychiat Res. 2017;256:207-11.

70. Yeragani VK, PohI R, Balon R, Ramesh C, Glitz D, Jung I, et al. Heart rate variability in patients with major depression. Psychiat Res. 1991;37(1):35-46.

71. Woody ML, McGeary JE, Gibb BE. Brooding Rumination and Heart Rate Variability in Women at High and Low Risk for Depression: Group Differences and Moderation by COMT Genotype. Journal of Abnormal Psychology. 2014;123(1):61-7.

72. Chalmers JA, Heathers JAJ, Abbott MJ, Kemp AH, Quintana DS. Worry is associated with robust reductions in heart rate variability: a transdiagnostic study of anxiety psychopathology.(Report). BMC Psychology. 2016;4(1).

73. Ottaviani C, Shahabi L, Tarvainen M, Cook I, Abrams M, Shapiro D. Cognitive, behavioral, and autonomic correlates of mind wandering and perseverative cognition in major depression. Frontiers in Neuroscience. 2015;8(433).

74. Bailey T, Shahabi L, Tarvainen M, Shapiro D, Ottaviani C. Moderating effects of the valence of social interaction on the dysfunctional consequences of perseverative cognition: an ecological study in major depression and social anxiety disorder. Anxiety, Stress, \& Coping. 2019;32(2):179-95.

75. Ottaviani C. Brain-heart interaction in perseverative cognition. Psychophysiology. 2018;55(7):n/a-n/a.

76. Kemp AH, Brunoni AR, Santos IS, Nunes MA, Dantas EM, de Figueiredo RC, et al. Effects of depression, anxiety, comorbidity, and antidepressants on resting-state heart rate and its variability: An ELSA-Brasil cohort baseline study.(Report)(Author abstract). American Journal of Psychiatry. 2014;171(12):1328.

77. Makovac E, Meeten F, Watson DR, Herman A, Garfinkel SN, D. Critchley H, et al. Alterations in Amygdala-Prefrontal Functional Connectivity Account for Excessive Worry and Autonomic Dysregulation in Generalized Anxiety Disorder. Biological Psychiatry. 2016;80(10):786-95.

78. Sgoifo A, Carnevali L, Pico Alfonso MdlA, Amore M. Autonomic dysfunction and heart rate variability in depression. Stress. 2015;18(3):343-52.

79. Mulcahy JS, Larsson DEO, Garfinkel SN, Critchley HD. Heart rate variability as a biomarker in health and affective disorders: A perspective on neuroimaging studies. Neuroimage. 2019;202:116072.

80. Chang H-A, Chang C-C, Tzeng N-S, Kuo TBJ, Lu R-B, Huang S-Y. Generalized anxiety disorder, comorbid major depression and heart rate variability: a case-control study in taiwan. Psychiatry investigation. 2013;10(4):326-35. 
81. Pittig A, Arch JJ, Lam CWR, Craske MG. Heart rate and heart rate variability in panic, social anxiety, obsessive-compulsive, and generalized anxiety disorders at baseline and in response to relaxation and hyperventilation. Int J Psychophysiol. 2013;87(1):19-27.

82. Kemp AH, Quintana DS, Felmingham KL, Matthews S, Jelinek HF. Depression, Comorbid Anxiety Disorders, and Heart Rate Variability in Physically Healthy, Unmedicated Patients: Implications for Cardiovascular Risk (Autonomic Nervous System Function in Depression). Plos One. 2012;7(2):e30777.

83. Udupa K, Sathyaprabha TN, Thirthalli J, Kishore KR, Lavekar GS, Raju TR, et al. Alteration of cardiac autonomic functions in patients with major depression: A study using heart rate variability measures. Journal of Affective Disorders. 2007;100(1-3):137-41.

84. Shinba T. Altered autonomic activity and reactivity in depression revealed by heart-rate variability measurement during rest and task conditions. Psychiatry and Clinical Neurosciences. 2014;68(3):225-33.

85. Shinba T. Major depressive disorder and generalized anxiety disorder show different autonomic dysregulations revealed by heart-rate variability analysis in first-onset drug-naïve patients without comorbidity. Psychiatry and Clinical Neurosciences. 2017;71(2):135-45.

86. Campbell AA, Wisco BE, Silvia PJ, Gay NG. Resting respiratory sinus arrhythmia and posttraumatic stress disorder: A meta-analysis. Biol Psychol. 2019;144(125-135):125-35.

87. Bassett D. A literature review of heart rate variability in depressive and bipolar disorders. Australian \& New Zealand Journal of Psychiatry. 2015;50(6):511-9.

88. Licht C, de Geus E, Penninx B. Association between Anxiety Disorders and Heart Rate Variability in The Netherlands Study of Depression and Anxiety (NESDA). Psychosomatic Medicine. 2009;71(5):508-18.

89. Rottenberg J. Cardiac vagal control in depression: A critical analysis. Biol Psychol. 2007;74(2):20011.

90. Kemp AH, Quintana DS, Gray MA, Felmingham KL, Brown K, Gatt JM. Impact of Depression and Antidepressant Treatment on Heart Rate Variability: A Review and Meta-Analysis. Biological Psychiatry. 2010;67(11):1067-74.

91. Alvares GA, Quintana DS, Kemp AH, Van Zwieten A, Balleine BW, Hickie IB, et al. Reduced Heart Rate Variability in Social Anxiety Disorder: Associations with Gender and Symptom Severity. Plos One. 2013;8(7):e70468.

92. Bassett D, Bear N, Nutt D, Hood S, Bassett S, Hans D. Reduced heart rate variability in remitted bipolar disorder and recurrent depression. Australian \& New Zealand Journal of Psychiatry. 2016;50(8):793804.

93. Lesnewich LM, Conway FN, Buckman JF, Brush CJ, Ehmann PJ, Eddie D, et al. Associations of depression severity with heart rate and heart rate variability in young adults across normative and clinical populations. Int J Psychophysiol. 2019;142:57-65. 
94. Huang M, Shah A, Su S, Goldberg J, Lampert RJ, Levantsevych OM, et al. Association of Depressive Symptoms and Heart Rate Variability in Vietnam War-Era Twins: A Longitudinal Twin Difference Study. JAMA Psychiatry. 2018;75(7):705-12.

95. Miller BJ, Paschall CB, Svendsen DP. Mortality and medical comorbidity among patients with serious mental illness. Psychiatric services (Washington, DC). 2006;57(10):1482.

96. Hert M, Correll CU, Bobes J, Cetkovich-Bakmas M, Cohen D, Asai I, et al. Physical illness in patients with severe mental disorders. I. Prevalence, impact of medications and disparities in health care. World psychiatry : official journal of the World Psychiatric Association (WPA). 2011;10(1):52-77.

97. Pruneti C, Cosentino C, Sgromo M, Innocenti A. Skin Conductance Response as a decisive variable in individuals with a DSM-IV TR Axis I diagnosis. JMED Res. 2014;565009(10.5171):2014.565009.

98. Vahey R, Becerra R. Galvanic Skin Response in Mood Disorders: A Critical Review. International journal of psychology and psychological therapy. 2015;15(2):275-304.

99. Pruneti C, Sacco M, Cosentino C, Sgromo D. Relevance of Autonomic Arousal in the Stress Response in Psychopathology. Journal of Basic \& Applied Sciences. 2016;12.

100. Iacono WG, Lykken DT, Peloquin LJ, Lumry AE, Valentine RH, Tuason VB. Electrodermal activity in euthymic unipolar and bipolar affective disorders. A possible marker for depression. Archives of general psychiatry. 1983;40(5):557-65.

101. Birket-Smith $\mathrm{M}$, Hasle N, Jensen $\mathrm{HH}$. Electrodermal activity in anxiety disorders. Acta Psychiatr Scand. 1993;88(5):350-5.

102. Kim AY, Jang EH, Kim S, Choi KW, Jeon HJ, Yu HY, et al. Automatic detection of major depressive disorder using electrodermal activity. Scientific Reports. 2018;8(1):17030.

103. Sarchiapone M, Gramaglia C, losue M, Carli V, Mandelli L, Serretti A, et al. The association between electrodermal activity (EDA), depression and suicidal behaviour: A systematic review and narrative synthesis. BMC Psychiatry. 2018;18(1):22.

104. Niv S. Clinical efficacy and potential mechanisms of neurofeedback. Pers Indiv Differ. 2013;54(6):676.

105. Menon B. Towards a new model of understanding - The triple network, psychopathology and the structure of the mind. Medical hypotheses. 2019;133:109385-.

106. Menon V. Brain networks and cognitive impairment in psychiatric disorders. World psychiatry : official journal of the World Psychiatric Association (WPA). 2020;19(3):309-10.

107. Janiri D, Moser DA, Doucet GE, Luber MJ, Rasgon A, Lee WH, et al. Shared Neural Phenotypes for Mood and Anxiety Disorders: A Meta-analysis of 226 Task-Related Functional Imaging Studies. JAMA Psychiatry. 2020;77(2):172-9.

108. Sha Z, Wager TD, Mechelli A, He Y. Common Dysfunction of Large-Scale Neurocognitive Networks Across Psychiatric Disorders. Biological Psychiatry. 2019;85(5):379-88.

109. Rayner G, Jackson G, Wilson S. Cognition-related brain networks underpin the symptoms of unipolar depression: Evidence from a systematic review. Neuroscience and Biobehavioral Reviews. 
2016;61:53-65.

110. Yang X, Liu J, Meng Y, Xia M, Cui Z, Wu X, et al. Network analysis reveals disrupted functional brain circuitry in drug-naive social anxiety disorder. Neuroimage. 2019;190:213-23.

111. Vanes L, Dolan R. Transdiagnostic neuroimaging markers of psychiatric risk: a narrative review. 2020.

112. Mulders PCR. Current Connections: Brain patterns in Electroconvulsive Therapy. 2020.

113. Zilcha-Mano S, Zhu X, Suarez-Jimenez B, Pickover A, Tal S, Such S, et al. Diagnostic and Predictive Neuroimaging Biomarkers for Posttraumatic Stress Disorder. Biological Psychiatry: Cognitive Neuroscience and Neuroimaging. 2020.

114. Bell AN, Moss D, Kallmeyer RJ. Healing the neurophysiological roots of trauma: A controlled study examining loreta z-score neurofeedback and HRV biofeedback for chronic PTSD. NeuroRegulation. 2019;6(2):54-70.

115. Vanhatalo S, Voipio J, Kaila K. Full-Band EEG (FbEEG): A New Standard for Clinical Electroencephalography. Clinical EEG and Neuroscience. 2005;36(4):311-7.

116. Aladjalova N, Kol'tsova A. Very slow rhythmic variations in the potential of the nuclei of the hypothalamus and the thalamus. Bulletin of Experimental Biology and Medicine. 1958;46(4):1153-7.

117. Aladjalova NA. Infra-Slow Rhythmic Oscillations of The Steady Potential of the Cerebral Cortex. Nature. 1957;179(4567):957-9.

118. Aladjalova NA. Slow electrical processes in the brain. Amsterdam, New York: Elsevier Pub. co.; 1964.

119. Vanhatalo S, Palva JM, Holmes MD, Miller JW, Voipio J, Kaila K. Infraslow oscillations modulate excitability and interictal epileptic activity in the human cortex during sleep. P Natl Acad Sci USA. 2004;101(14):5053-7.

120. He BJ, Raichle ME. The fMRI signal, slow cortical potential and consciousness. 2009. p. 302-9.

121. Niedermeyer E, Schomer DL, Lopes da Silva FH. Niedermeyer's electroencephalography : basic principles, clinical applications, and related fields. Sixth edition. ed. Philadelphia: Wolters Kluwer/Lippincott Williams \& Wilkins Health; 2011.

122. Smith M. Infra-slow fluctuation training; On the down-low in neuromodulation2013.

123. He BJ, Snyder AZ, Zempel JM, Smyth MD, Raichle ME. Electrophysiological correlates of the brain's intrinsic large-scale functional architecture. P Natl Acad Sci USA. 2008;105(41):16039.

124. Khader P, Schicke T, Röder B, Rösler F. On the relationship between slow cortical potentials and BOLD signal changes in humans. Int J Psychophysiol. 2008;67(3):252-61.

125. Pan WJ, Thompson GJ, Magnuson ME, Jaeger D, Keilholz S. Infraslow LFP correlates to resting-state fMRI BOLD signals. Neuroimage. 2013;74:288-97.

126. Leistner HS, Sander HT, Wuebbeler HG, Link HA, Elster HC, Curio HG, et al. Magnetoencephalography discriminates modality-specific infraslow signals less than 0.1 Hz. NeuroReport. 2010;21(3):196-200.

127. Krishnan G, González O, Bazhenov M. Origin of slow spontaneous resting-state neuronal fluctuations in brain networks. P Natl Acad Sci USA. 2018;115(26):6858. 
128. Watson BO. Cognitive and Physiologic Impacts of the Infraslow Oscillation. Frontiers in Systems Neuroscience. 2018;12.

129. Timofeev I, Grenier F, Bazhenov M, Sejnowski T, Steriade M. Origin of Slow Cortical Oscillations in Deafferented Cortical Slabs. Cerebral Cortex. 2000;10(12):1185.

130. Lőrincz ML, Geall F, Bao Y, Crunelli V, Hughes SW. ATP-Dependent Infra-Slow $(<0.1 \mathrm{~Hz})$ Oscillations in Thalamic Networks (Infra-Slow Oscillations). Plos One. 2009;4(2):e4447.

131. Hughes SW, Lőrincz ML, Parri HR, Crunelli V. Infraslow $(<0.1 \mathrm{~Hz})$ oscillations in thalamic relay nuclei: basic mechanisms and significance to health and disease states. Progress in Brain Research. 2011;193:145-62.

132. van Putten MJAM, Tjepkema-Cloostermans MC, Hofmeijer J. Infraslow EEG activity modulates cortical excitability in postanoxic encephalopathy. Journal of neurophysiology. 2015;113(9):3256-67.

133. Filippov IV, Williams WC, Frolov VA. Very slow potential oscillations in locus coeruleus and dorsal raphe nucleus under different illumination in freely moving rats. Neuroscience Letters. 2004;363(1):89-93.

134. Dash MB, Ajayi S, Folsom L, Gold PE, Korol DL. Spontaneous Infraslow Fluctuations Modulate Hippocampal EPSP-PS Coupling. eNeuro. 2018;5(1).

135. Abbas A, Belloy M, Kashyap A, Billings J, Nezafati M, Schumacher EH, et al. Quasi-periodic patterns contribute to functional connectivity in the brain. Neuroimage. 2019;191:193-204.

136. Grooms JK, Thompson GJ, Pan WJ, Billings J, Schumacher EH, Epstein CM, et al. Infraslow Electroencephalographic and Dynamic Resting State Network Activity. Brain Connect. 2017;7(5):26580.

137. Buzsáki G, Draguhn A. Neuronal oscillations in cortical networks. Science (New York, NY). 2004;304(5679):1926-9.

138. Palva JM, Palva S. Roles of multiscale brain activity fluctuations in shaping the variability and dynamics of psychophysical performance. Progress in Brain Research. 2011;193:335-50.

139. Palva JM, Palva S. Infra-slow fluctuations in electrophysiological recordings, blood-oxygenationlevel-dependent signals, and psychophysical time series. Neuroimage. 2012;62(4):2201-11.

140. Engel Andreas k, Gerloff C, Hilgetag Claus c, Nolte G. Intrinsic Coupling Modes: Multiscale Interactions in Ongoing Brain Activity. Neuron. 2013;80(4):867-86.

141. Weaver KE, Wander JD, Ko AL, Casimo K, Grabowski TJ, Ojemann JG, et al. Directional patterns of cross frequency phase and amplitude coupling within the resting state mimic patterns of $\mathrm{FMRI}$ functional connectivity. Neuroimage. 2016;128:238-51.

142. Florin $E$, Baillet $S$. The brain's resting-state activity is shaped by synchronized cross-frequency coupling of neural oscillations. Neuroimage. 2015;111:26-35.

143. Wang L, Saalmann Yuri b, Pinsk Mark a, Arcaro Michael j, Kastner S. Electrophysiological LowFrequency Coherence and Cross-Frequency Coupling Contribute to BOLD Connectivity. Neuron. 2012;76(5):1010-20. 
144. Cannon R, Congedo M, Lubar J, Hutchens T. Differentiating a Network of Executive Attention: Loreta Neurofeedback in Anterior Cingulate and Dorsolateral Prefrontal Cortices. Int J Neurosci. 2009;119(3):404-41.

145. Vaitl D, Birbaumer N, Gruzelier J, Jamieson GA, Kotchoubey B, Kubler A, et al. Psychobiology of altered states of consciousness. Psychol Bull. 2005;131(1):98-127.

146. Imperatori C, Valenti EM, Della Marca G, Amoroso N, Massullo C, Carbone GA, et al. Coping food craving with neurofeedback. Evaluation of the usefulness of alpha/theta training in a non-clinical sample. Int J Psychophysiol. 2017;112:89-97.

147. Kluetsch RC, Ros T, Theberge J, Frewen PA, Calhoun VD, Schmahl C, et al. Plastic modulation of PTSD resting-state networks and subjective wellbeing by EEG neurofeedback. Acta Psychiat Scand. 2014;130(2):123-36.

148. Lackner N, Unterrainer HF, Skliris D, Shaheen S, Dunitz-Scheer M, Wood G, et al. EEG neurofeedback effects in the treatment of adolescent anorexia nervosa. Eat Disord. 2016;24(4):354-74.

149. Raymond J, Varney C, Parkinson LA, Gruzelier JH. The effects of alpha/theta neurofeedback on personality and mood. Cognitive Brain Res. 2005;23(2-3):287-92.

150. Trudeau DL. EEG biofeedback for addictive disorders - The state of the art in 2004. J Adult Dev. 2005;12(2-3):139-46.

151. Fragedakis TM, Toriello P. The Development and Experience of Combat-Related PTSD: A Demand for Neurofeedback as an Effective Form of Treatment. Journal of Counseling \& Development. 2014;92(4):481-8.

152. Peniston EG, Kulkosky PJ. Alpha-theta brainwave neurofeedback for Vietnam veterans with combatrelated post-traumatic stress disorder. Medical Psychotherapy. 1991;4(1):47-60.

153. Kerson C, Sherman RA, Kozlowski GP. Alpha suppression and symmetry training for generalized anxiety symptoms. Journal of Neurotherapy. 2009;13(3):146-55.

154. Cheon EJ, Choi JH, Lee GW, Koo BH, Seo WS, Kim HG, et al. P.2.f.009 - Neurofeedback treatment on depressive symptoms and functional recovery and brain-derived neurotrophic factor in treatmentresistant major depression. European Neuropsychopharmacology. 2017;27:S851.

155. Liu H. Neurofeedback Training Intervention for Persons with Major Depression Disorder: Reducing Depressive Symptoms. NeuroQuantology. 2017;15(3).

156. Wang S-Y, Lin IM, Fan S-Y, Tsai Y-C, Yen C-F, Yeh Y-C, et al. The effects of alpha asymmetry and highbeta down-training neurofeedback for patients with the major depressive disorder and anxiety symptoms. Journal of Affective Disorders. 2019;257:287-96.

157. Chiba T, Kanazawa T, Koizumi A, Ide K, Taschereau-Dumouchel V, Boku S, et al. Current Status of Neurofeedback for Post-traumatic Stress Disorder: A Systematic Review and the Possibility of Decoded Neurofeedback.(Report). Frontiers in Human Neuroscience. 2019;13.

158. Gapen M, van der Kolk BA, Hamlin E, Hirshberg L, Suvak M, Spinazzola J. A Pilot Study of Neurofeedback for Chronic PTSD. Applied Psychophysiology Biofeedback. 2016;41(3):251-61. 
159. Chrapusta A, Pąchalska M, Wilk-Frańczuk M, Starczyńska M, Kropotov JD. Evaluation of the effectiveness of neurofeedback in the reduction of Posttraumatic stress disorder (PTSD) in a patient following high-voltage electric shock with the use of ERPs. Annals of Agricultural and Environmental Medicine. 2015;22(3):556-63.

160. Van Der Kolk BA, Hodgdon H, Gapen M, Musicaro R, Suvak MK, Hamlin E, et al. A randomized controlled study of neurofeedback for chronic PTSD. Plos One. 2016;11(12).

161. Wang SY, Lin IM, Peper E, Chen YT, Tang TC, Yeh YC, et al. The efficacy of neurofeedback among patients with major depressive disorder: Preliminary study. NeuroRegulation. 2016;3(3):127-34.

162. Cheon E-J, Koo B-H, Choi J-H. The Efficacy of Neurofeedback in Patients with Major Depressive Disorder: An Open Labeled Prospective Study. Applied Psychophysiology and Biofeedback. 2016;41(1):103-10.

163. Sadjadi SA, Hashemian P. Effectiveness of neurofeedback therapy in children with separation anxiety disorder. African Journal of Psychiatry (South Africa). 2014;17(6).

164. Noohi S, Miraghaie AM, Arabi A. Effectiveness of neuro-feedback treatment with alpha/theta method on PTSD symptoms and their executing function. Biomedical Research (India). 2017;28(5):2019-27.

165. Askovic M, Watters AJ, Aroche J, Harris AW. Neurofeedback as an adjunct therapy for treatment of chronic posttraumatic stress disorder related to refugee trauma and torture experiences: Two case studies. Australasian Psychiatry. 2017;25(4):358-63.

166. Askovic M, Watters AJ, Coello M, Aroche J, Harris AWF, Kropotov J. Evaluation of Neurofeedback for Posttraumatic Stress Disorder Related to Refugee Experiences Using Self-Report and Cognitive ERP Measures. Clinical EEG and Neuroscience. 2019.

167. Choi SW, Chi SE, Chung SY, Kim JW, Ahn CY, Kim HT. Is Alpha Wave Neurofeedback Effective with Randomized Clinical Trials in Depression? A Pilot Study. Neuropsychobiology. 2010;63(1):43-51.

168. Dias AM, van Deusen A. A new neurofeedback protocol for depression. The Spanish journal of psychology. 2011;14(1):374-84.

169. Hammond D. Neurofeedback Treatment of Depression and Anxiety. J Adult Dev. 2005;12(2-3):131-7.

170. Hammond DC. QEEG-guided neurofeedback in the treatment of obsessive compulsive disorder. Journal of Neurotherapy. 2003;7(2):25-52.

171. Cheon E-J, Koo B-H, Seo W-S, Lee J-Y, Choi J-H, Song S-H. Effects of Neurofeedback on Adult Patients with Psychiatric Disorders in a Naturalistic Setting. Applied Psychophysiology and Biofeedback. 2015;40(1):17-24.

172. Kluetsch RC, Ros T, Théberge J, Frewen PA, Calhoun VD, Schmahl C, et al. Plastic modulation of PTSD resting-state networks and subjective wellbeing by EEG neurofeedback. Acta Psychiat Scand. 2014;130(2):123-36.

173. Zhang P, Cheng L. A Randomized Controlled Trial of a Neurofeedback-based Training for Improvement in Social Phobia Disorder. NeuroQuantology. 2017;15(4). 
174. Peeters F, Oehlen M, Ronner J, van Os J, Lousberg R. Neurofeedback As a Treatment for Major Depressive Disorder - A Pilot Study. Plos One. 2014;9(3):e91837.

175. Lu Y, Wang C, Su L, Ma Z, Li S, Fan Y. Effects of Neurofeedback on Panic Disorder Patients' anxiety. NeuroQuantology. 2017;15(3).

176. Ros T, Frewen P, Théberge J, Michela A, Kluetsch R, Mueller A, et al. Neurofeedback Tunes Scale-Free Dynamics in Spontaneous Brain Activity. Cerebral Cortex. 2017;27(10):4911-22.

177. Escolano C, Navarro-Gil M, Garcia-Campayo J, Congedo M, De Ridder D, Minguez J. A controlled study on the cognitive effect of alpha neurofeedback training in patients with major depressive disorder. Frontiers in Behavioral Neuroscience. 2014;8:296.

178. Orndorff-Plunkett F, Singh F, Aragón O, Pineda J. Assessing the Effectiveness of Neurofeedback Training in the Context of Clinical and Social Neuroscience. Brain Sciences. 2017;7(8):95.

179. Reiter BK, Andersen BS, Carlsson BJ. Neurofeedback Treatment and Posttraumatic Stress Disorder: Effectiveness of Neurofeedback on Posttraumatic Stress Disorder and the Optimal Choice of Protocol. The Journal of Nervous and Mental Disease. 2016;204(2):69-77.

180. Simkin DR, Thatcher RW, Lubar J. Quantitative EEG and Neurofeedback in Children and Adolescents: Anxiety Disorders, Depressive Disorders, Comorbid Addiction/ADHD, and Brain Injury. Child and Adolescent Psychiatric Clinics of North America. 2014;23(3).

181. Rance M, Walsh C, Sukhodolsky DG, Pittman B, Qiu M, Kichuk SA, et al. Time course of clinical change following neurofeedback. Neuroimage. 2018;181:807-13.

182. Schabus M, Griessenberger H, Gnjezda MT, Heib DPJ, Wislowska M, Hoedlmoser K. Better than sham? A double-blind placebo-controlled neurofeedback study in primary insomnia. Brain. 2017;140(4):1041-52.

183. Thibault RT, Raz A. Neurofeedback: The power of psychosocial therapeutics. The Lancet Psychiatry. 2016;3(11):e18.

184. Thibault RT, Lifshitz M, Raz A. The climate of neurofeedback: scientific rigour and the perils of ideology. Brain. 2018;141(2):e11-e.

185. Thibault RT, Raz A. When can neurofeedback join the clinical armamentarium? The Lancet Psychiatry. 2016;3(6):497-8.

186. Thibault RT, Lifshitz M, Birbaumer N, Raz A. Neurofeedback, Self-Regulation, and Brain Imaging: Clinical Science and Fad in the Service of Mental Disorders. Psychother Psychosom. 2015;84(4):193207.

187. Thibault RT, Lifshitz M, Raz A. The self-regulating brain and neurofeedback: Experimental science and clinical promise. Cortex. 2016;74:247-61.

188. Thibault RT, Lifshitz M, Raz A. Neurofeedback or neuroplacebo? Brain. 2017;140(4):862-4.

189. Thibault RT, Raz A. The Psychology of Neurofeedback: Clinical Intervention Even if Applied Placebo. American Psychologist. 2017;72(7):679-88. 
190. Schönenberg M, Wiedemann E, Schneidt A, Scheeff J, Logemann A, Keune PM, et al. Neurofeedback, sham neurofeedback, and cognitive-behavioural group therapy in adults with attention-deficit hyperactivity disorder: a triple-blind, randomised, controlled trial. The Lancet Psychiatry. 2017;4(9):673-84.

191. Schönenberg M, Wiedemann E, Schneidt A, Scheeff J, Logemann A, Keune PM, et al. Confusion regarding operant conditioning of the EEG - Authors' reply. In: Schönenberg M, editor. 2017. p. 897-8.

192. Arnold LE, Arns M, Barterian J, Bergman R, Black S, Conners CK, et al. Double-Blind PlaceboControlled Randomized Clinical Trial of Neurofeedback for Attention-Deficit/Hyperactivity Disorder With 13 Month Follow-up. Journal of the American Academy of Child \& Adolescent Psychiatry. 2020.

193. Pascual-Marqui RD. Standardized low resolution brain electromagnetic tomography (SLORETA): Technical details. Methods and findings in experimental and clinical pharmacology. 2002;24 Suppl D:5-12.

194. Leong SL, Vanneste S, Lim J, Smith M, Manning P, De Ridder D. A randomised, double-blind, placebocontrolled parallel trial of closed-loop infraslow brain training in food addiction. Scientific Reports. 2018;8(1).

195. Leong SL, Vanneste S, Lim J, Smith M, Manning P, De Ridder D, et al. Effects and Side Effects of Infraslow Network Neurofeedback (ISF-NF): a randomised double blind placebo controlled trial. Int J Psychophysiol. 2018;131:S151.

196. Goldstein-Piekarski AN, Williams LM, Humphreys K. A trans-diagnostic review of anxiety disorder comorbidity and the impact of multiple exclusion criteria on studying clinical outcomes in anxiety disorders. Translational Psychiatry. 2016;6(6):e847.

197. Ehring T, Watkins E. Repetitive Negative Thinking as a Transdiagnostic Process. International Journal of Cognitive Therapy - INT J COGN THER. 2008;1:192-205.

198. Epkins C, Heckler D. Integrating Etiological Models of Social Anxiety and Depression in Youth: Evidence for a Cumulative Interpersonal Risk Model. Clinical Child and Family Psychology Review. 2011;14(4):329-76.

199. Cummings CM, Caporino NE, Kendall PC. Comorbidity of Anxiety and Depression in Children and Adolescents: 20 Years After. Psychol Bull. 2014;140(3):816-45.

200. Lahey BB, Krueger RF, Rathouz PJ, Waldman ID, Zald DH. A Hierarchical Causal Taxonomy of Psychopathology Across the Life Span. Psychol Bull. 2017;143(2):142-86.

201. Zald DH, Lahey BB. Implications of the Hierarchical Structure of Psychopathology for Psychiatric Neuroimaging. Biological Psychiatry: Cognitive Neuroscience and Neuroimaging. 2017;2(4):310-7.

202. Dickinson D. "If the Shoe Fits ...": The Hierarchical Structure of Psychopathology and Psychiatric Neuroimaging. Biological Psychiatry: Cognitive Neuroscience and Neuroimaging. 2017;2(4):303-4.

203. Bui E, Fava M. From depression to anxiety, and back. Acta Psychiat Scand. 2017;136(4):341-2.

204. Wenzel A, Jager-Hyman S. Chapter 9 - Social Anxiety Disorder and Its Relation to Clinical Syndromes in Adulthood. In: Hofmann SG, DiBartolo PM, editors. Social Anxiety (Third Edition). San Diego: Academic Press; 2014. p. 227-51. 
205. Scholten WD, Batelaan NM, Penninx BWJH, Balkom AJLMv, Smit JH, Schoevers RA, et al. Diagnostic instability of recurrence and the impact on recurrence rates in depressive and anxiety disorders. Journal of Affective Disorders. 2016;195:185-90.

206. McTeague LM, Goodkind MS, Etkin A. Transdiagnostic impairment of cognitive control in mental illness. Journal of Psychiatric Research. 2016;83:37-46.

207. Verduin TL, Kendall PC. Differential Occurrence of Comorbidity Within Childhood Anxiety Disorders. Journal of Clinical Child \& Adolescent Psychology. 2003;32(2):290-5.

208. Koyuncu A, Ertekin E, Binbay Z, Özyıldırım I, Yüksel Ç, Tükel R. The clinical impact of mood disorder comorbidity on social anxiety disorder. Comprehensive Psychiatry. 2014;55(2):363-9.

209. Schneier FR, Johnson J, Hornig CD, Liebowitz MR, Weissman MM. Social phobia. Comorbidity and morbidity in an epidemiologic sample. Archives of general psychiatry. 1992;49(4):282.

210. Lampe L, Slade T, Issakidis C, Andrews G. Social phobia in the Australian National Survey of Mental Health and Well-Being (NSMHWB). Psychological Medicine. 2003;33(4):637-46.

211. Goldberg D, Fawcett J. THE IMPORTANCE OF ANXIETY IN BOTH MAJOR DEPRESSION AND BIPOLAR DISORDER. 2012. p. 471-8.

212. Verduijn J, Verhoeven JE, Milaneschi Y, Schoevers RA, van Hemert AM, Beekman ATF, et al. Reconsidering the prognosis of major depressive disorder across diagnostic boundaries: Full recovery is the exception rather than the rule. BMC medicine. 2017;15(1):urn:issn:1741-7015.

213. Brown TA, Campbell LA, Lehman CL, Grisham JR, Mancill RB. Current and Lifetime Comorbidity of the DSM-IV Anxiety and Mood Disorders in a Large Clinical Sample. Journal of Abnormal Psychology. 2001;110(4):585-99.

214. Richards D. Prevalence and clinical course of depression: A review. Clinical Psychology Review. 2011;31(7):1117-25.

215. Belzer RK, Schneier RF. Comorbidity of Anxiety and Depressive Disorders: Issues in Conceptualization, Assessment, and Treatment. Journal of Psychiatric Practice. 2004;10(5):296-306.

216. Slade TIM, Watson D. The structure of common DSM-IV and ICD-10 mental disorders in the Australian general population. Psychological Medicine. 2006;36(11):1593-600.

217. Grant BF, Hasin DS, Stinson FS, Dawson DA, June Ruan W, Goldstein RB, et al. Prevalence, correlates, co-morbidity, and comparative disability of DSM-IV generalized anxiety disorder in the USA: results from the National Epidemiologic Survey on Alcohol and Related Conditions. Psychological Medicine. 2005;35(12):1747-59.

218. Gorman JM. Comorbid depression and anxiety spectrum disorders. Depression and Anxiety. 1996;4(4):160-8.

219. Mennin DS, Heimberg RG, Jack MS. Comorbid Generalized Anxiety Disorder in Primary Social Phobia: Symptom Severity, Functional Impairment, and Treatment Response. Journal of Anxiety Disorders. 2000;14(4):325-43.

220. Tiller JW. Depression and anxiety. The Medical journal of Australia. 2013;199(6 Suppl):S28-31. 
221. Yonkers KA, Bruce SE, Dyck IR, Keller MB. Chronicity, relapse, and illness-course of panic disorder, social phobia, and generalized anxiety disorder: Findings in men and women from 8 years of followup. Depression and Anxiety. 2003;17(3):173-9.

222. Simon NM. Generalized Anxiety Disorder and Psychiatric Comorbidities Such as Depression, Bipolar Disorder, and Substance Abuse. The Journal of Clinical Psychiatry. 2009;70(suppl 2):10-4.

223. Hendriks SM, Spijker J, Licht CMM, Beekman ATF, Penninx BWJH. Two-year course of anxiety disorders: different across disorders or dimensions? Acta Psychiat Scand. 2013;128(3):212-21.

224. Wu Z, Fang Y. Comorbidity of depressive and anxiety disorders: challenges in diagnosis and assessment. Shanghai archives of psychiatry. 2014;26(4):227-31.

225. Lamers F, van Oppen P, Comijs HC, Smit JH, Spinhoven P, van Balkom AJLM, et al. Comorbidity patterns of anxiety and depressive disorders in a large cohort study: the Netherlands Study of Depression and Anxiety (NESDA). The Journal of clinical psychiatry. 2011;72(3):341-8.

226. Garber J, Weersing VR. Comorbidity of Anxiety and Depression in Youth: Implications for Treatment and Prevention. Oxford, UK2010. p. 293-306.

227. Stein MB, Sareen J. Generalized Anxiety Disorder. New England Journal of Medicine. 2015;373(21):2059-68.

228. Sunderland M, Mewton L, Slade T, Baillie AJ. Investigating differential symptom profiles in major depressive episode with and without generalized anxiety disorder: true co-morbidity or symptom similarity? Psychological Medicine. 2010;40(7):1113-23.

229. American Psychiatric A, American Psychiatric Association DSMTF. Diagnostic and statistical manual of mental disorders : DSM-5. 5th ed. ed. Washington, D.C.: American Psychiatric Publishing; 2013.

230. Penninx BWJH, Nolen WA, Lamers F, Zitman FG, Smit JH, Spinhoven P, et al. Two-year course of depressive and anxiety disorders: Results from the Netherlands Study of Depression and Anxiety (NESDA). Journal of Affective Disorders. 2011;133:76-85.

231. Boschloo L, Schoevers RA, Beekman ATF, Smit JH, van Hemert AM, Penninx BWJH. The Four-Year Course of Major Depressive Disorder: The Role of Staging and Risk Factor Determination. Psychother Psychosom. 2014;83(5):279-88.

232. Moffitt TE, Harrington H, Caspi A, Kim-Cohen J, Goldberg D, Gregory AM, et al. Depression and generalized anxiety disorder: cumulative and sequential comorbidity in a birth cohort followed prospectively to age 32 years. Archives of general psychiatry. 2007;64(6):651.

233. Beesdo K, Bittner A, Pine DS, Stein MB, Höfler M, Lieb R, et al. Incidence of social anxiety disorder and the consistent risk for secondary depression in the first three decades of life. Archives of general psychiatry. 2007;64(8):903.

234. Kessler RC, Chiu WT, Demler O, Walters EE. Prevalence, severity, and comorbidity of 12-month DSM-IV disorders in the National Comorbidity Survey Replication.(Original Article)(Diagnostic and Statistical Manual of Mental Disorders). Archives of General Psychiatry. 2005;62(6):617.

235. Brockveld KC, Perini SJ, Rapee RM. Chapter 6 - Social Anxiety and Social Anxiety Disorder Across Cultures. In: Hofmann SG, DiBartolo PM, editors. Social Anxiety (Third Edition). San Diego: Academic 
Press; 2014. p. 141-58.

236. Newman DL, Moffitt TE, Caspi A, Magdol L, Silva PA, Stanton WR. Psychiatric Disorder in a Birth Cohort of Young Adults: Prevalence, Comorbidity, Clinical Significance, and New Case Incidence From Ages 11 to 21. Journal of Consulting and Clinical Psychology. 1996;64(3):552-62.

237. Kessler RC, Berglund P, Demler O, Jin R, Merikangas KR, Walters EE. Lifetime prevalence and age-ofonset distributions of DSM-IV disorders in the National Comorbidity Survey Replication. Archives of general psychiatry. 2005;62(6):593.

238. Bruce SE, Yonkers KA, Otto MW, Eisen JL, Weisberg RB, Pagano M, et al. Influence of psychiatric comorbidity on recovery and recurrence in generalized anxiety disorder, social phobia, and panic disorder: a 12-year prospective study. The American journal of psychiatry. 2005;162(6):1179.

239. Kessler RC. The epidemiology of pure and comorbid generalized anxiety disorder: a review and evaluation of recent research. In: Kessler RC, editor. 2000. p. 7-13.

240. Zbozinek TD, Rose RD, Wolitzky-Taylor KB, Sherbourne C, Sullivan G, Stein MB, et al. Diagnostic overlap of generalized anxiety disorder and major depressive disorder in a primary care sample. Depression and anxiety. 2012;29(12):1065-71.

241. Schwartz S, Susser E. The use of well controls: an unhealthy practice in psychiatric research. Psychological Medicine. 2011;41(6):1127-31.

242. Begemann MJ, Florisse EJ, van Lutterveld R, Kooyman M, Sommer IE. Efficacy of EEG neurofeedback in psychiatry: A comprehensive overview and meta-analysis. Translational Brain Rhythmicity. 2016;1(1):19-29.

243. Arns M, Batail JM, Bioulac S, Congedo M, Daudet C, Drapier D, et al. Neurofeedback: One of today's techniques in psychiatry? L'Encéphale. 2017;43(2):135-45.

244. Omejc N, Rojc B, Battaglini PP, Marusic U. Review of the therapeutic neurofeedback method using electroencephalography: EEG Neurofeedback. Bosnian journal of basic medical sciences. 2019;19(3):213.

245. Ross S, Grant A, Counsell C, Gillespie W, Russell I, Prescott R. Barriers to participation in randomised controlled trials: a systematic review. J Clin Epidemiol. 1999;52(12):1143-56.

246. Scharnowski F, Veit R, Zopf R, Studer P, Bock S, Diedrichsen J, et al. Manipulating motor performance and memory through real-time fMRI neurofeedback. Biol Psychol. 2015;108:85-97.

247. Sepulveda P, Sitaram R, Rana M, Montalba C, Tejos C, Ruiz S. How feedback, motor imagery, and reward influence brain self-regulation using real-time fMRI. Hum Brain Mapp. 2016;37(9):3153-71.

248. Strehl U. What learning theories can teach us in designing neurofeedback treatments. Frontiers in human neuroscience. 2014;8:894.

249. Kober S, Witte M, Ninaus M, Neuper C, Wood G. Learning to modulate one's own brain activity: the effect of spontaneous mental strategies. Frontiers in Human Neuroscience. 2013;7:695.

250. Micoulaud-Franchi JA, McGonigal A, Lopez R, Daudet C, Kotwas I, Bartolomei F.

Electroencephalographic neurofeedback: Level of evidence in mental and brain disorders and 
suggestions for good clinical practice. Neurophysiologie Clinique/Clinical Neurophysiology. 2015;45(6):423-33.

251. Gruzelier JH. EEG-neurofeedback for optimising performance. III: A review of methodological and theoretical considerations. Neuroscience and Biobehavioral Reviews. 2014;44:159.

252. Sulzer J, Haller S, Scharnowski F, Weiskopf N, Birbaumer N, Blefari ML, et al. Real-time fMRI neurofeedback: Progress and challenges. Neuroimage. 2013;76(1):386-99.

253. Vanneste S, Joos K, Ost J, De Ridder D. Influencing connectivity and cross-frequency coupling by real-time source localized neurofeedback of the posterior cingulate cortex reduces tinnitus related distress. Neurobiology of stress. 2018;8:211-24.

254. Sherlin LH, Arns M, Lubar J, Heinrich H, Kerson C, Strehl U, et al. Neurofeedback and Basic Learning Theory: Implications for Research and Practice. Journal of Neurotherapy. 2011;15(4):292-304.

255. Audacity. 2.3.2 ed1999-2020.

256. Cannon R. LORETA Neurofeedback: Odd Reports, Observations, and Findings Associated with Spatial Specific Neurofeedback Training. Journal of Neurotherapy. 2012;16:164-7.

257. Hoffmann TC, Glasziou PP, Boutron I, Milne R, Perera R, Moher D, et al. Better reporting of interventions: template for intervention description and replication (TIDieR) checklist and guide. BMJ : British Medical Journal. 2014;348:g1687.

258. Health USDo, Human Services FDACfDE, Research, Health USDo, Human Services FDACfBE, Research, et al. Guidance for industry: patient-reported outcome measures: use in medical product development to support labeling claims: draft guidance. Health Qual Life Outcomes. 2006;4(1):79.

259. Doward LC, Gnanasakthy A, Baker MG. Patient reported outcomes: looking beyond the label claim. Health Qual Life Outcomes. 2010;8:89.

260. Watson D, O'hara MW, Naragon-Gainey K, Koffel E, Chmielewski M, Kotov R, et al. Development and Validation of New Anxiety and Bipolar Symptom Scales for an Expanded Version of the IDAS (the IDAS-II). Assessment. 2012;19(4):399-420.

261. Watson D, O'Hara MW. Understanding the Emotional DisordersA Symptom-Level Approach Based on the IDAS-II: A Symptom-Level Approach Based on the IDAS-II: Oxford University Press; 2017 2017-06.

262. Rosellini AJ, Brown TA. The Multidimensional Emotional Disorder Inventory (MEDI): Assessing Transdiagnostic Dimensions to Validate a Profile Approach to Emotional Disorder Classification. Psychological Assessment. 2019;31(1):59-72.

263. Zigmond AS, Snaith RP. The Hospital Anxiety and Depression Scale. Acta Psychiat Scand. 1983;67(6):361-70.

264. Carleton RN, Norton MAPJ, Asmundson GJG. Fearing the unknown: A short version of the Intolerance of Uncertainty Scale. Journal of Anxiety Disorders. 2007;21(1):105-17.

265. McEvoy PM, Mahoney AEJ, Moulds ML. Are worry, rumination, and post-event processing one and the same? Journal of Anxiety Disorders. 2010;24(5):509-19. 
266. McEvoy PM, Thibodeau MA, Asmundson GJG. Trait Repetitive Negative Thinking: A Brief Transdiagnostic Assessment. Journal of Experimental Psychopathology. 2014;5(3):jep.037813.

267. McEvoy PM, Hyett MP, Ehring T, Johnson SL, Samtani S, Anderson R, et al. Transdiagnostic assessment of repetitive negative thinking and responses to positive affect: Structure and predictive utility for depression, anxiety, and mania symptoms. Journal of Affective Disorders. 2018;232:37584.

268. Sheehan DV, Lecrubier Y, Sheehan KH, Amorim P, Janavs J, Weiller E, et al. The Mini-International Neuropsychiatric Interview (M.I.N.I.): the development and validation of a structured diagnostic psychiatric interview for DSM-IV and ICD-10. The Journal of clinical psychiatry. 1998;59 Suppl 20:22.

269. Lecrubier Y, Sheehan DV, Weiller E, Amorim P, Bonora I, Harnett Sheehan K, et al. The Mini International Neuropsychiatric Interview (MINI). A short diagnostic structured interview: reliability and validity according to the CIDI. European Psychiatry. 1997;12(5):224-31.

270. Sheehan DV, Lecrubier Y, Harnett Sheehan K, Janavs J, Weiller E, Keskiner A, et al. The validity of the Mini International Neuropsychiatric Interview (MINI) according to the SCID-P and its reliability. European Psychiatry. 1997;12(5):232-41.

271. Posner K, Brown, G. K., Stanley, B., Brent, D. A., Yershova, K. V., Oquendo, M. A., Currier, G. W., Melvin, G. A., Greenhill, L., Shen, S., and Mann J. J. The Columbia-Suicide Severity Rating Scale: Initial validity and internal consistency findings from three multisite studies with adolescents and adults. American Journal of Psychiatry. 2011;168(12):1266-77.

272. Shannon BJ, Dosenbach RA, Su Y, Vlassenko AG, Larson-Prior LJ, Nolan TS, et al. Morning-evening variation in human brain metabolism and memory circuits. Journal of neurophysiology. 2013;109(5):1444-56.

273. Lohmann G, Margulies DS, Horstmann A, Pleger B, Lepsien J, Goldhahn D, et al. Eigenvector Centrality Mapping for Analyzing Connectivity Patterns in fMRI Data of the Human Brain (Eigenvector Centrality Maps). Plos One. 2010;5(4):e10232.

274. Young HA, Cousins A, Johnston S, Fletcher JM, Benton D. Autonomic adaptations mediate the effect of hydration on brain functioning and mood: Evidence from two randomized controlled trials. Scientific Reports. 2019;9(1):16412.

275. Liao W, Fan Y-S, Yang S, Li J, Duan X, Cui Q, et al. Preservation Effect: Cigarette Smoking Acts on the Dynamic of Influences Among Unifying Neuropsychiatric Triple Networks in Schizophrenia. Schizophrenia bulletin. 2019;45(6):1242.

276. Jobert M, Wilson FJ, Ruigt GSF, Brunovsky M, Prichep LS, Drinkenburg WHIM. Guidelines for the Recording and Evaluation of Pharmaco-EEG Data in Man: The International Pharmaco-EEG Society (IPEG). Neuropsychobiology. 2012;66(4):201-20.

277. Shehzad Z, Kelly AMC, Reiss PT, Gee DG, Gotimer K, Uddin LQ, et al. The Resting Brain: Unconstrained yet Reliable. Cerebral Cortex. 2009;19(10):2209-29.

278. Noble S, Spann MN, Tokoglu F, Shen X, Constable RT, Scheinost D. Influences on the Test-Retest Reliability of Functional Connectivity MRI and its Relationship with Behavioral Utility. Cerebral Cortex. 
2017;27(11):5415-29.

279. Pannunzi M, Hindriks R, Bettinardi RG, Wenger E, Lisofsky N, Martensson J, et al. Resting-state fMRI correlations: From link-wise unreliability to whole brain stability. Neuroimage. 2017;157:250-62.

280. Gratton C, Laumann TO, Nielsen AN, Greene DJ, Gordon EM, Gilmore AW, et al. Functional Brain Networks Are Dominated by Stable Group and Individual Factors, Not Cognitive or Daily Variation. Neuron. 2018;98(2):439-52.e5.

281. Qualtrics. December, 2020 ed2005-2020.

282. Lane SJ, Heddle NM, Arnold E, Walker I. A review of randomized controlled trials comparing the effectiveness of hand held computers with paper methods for data collection. BMC medical informatics and decision making. 2006;6(1):23.

283. Dale 0 , Hagen KB. Despite technical problems personal digital assistants outperform pen and paper when collecting patient diary data. Journal of clinical epidemiology. 2007;60(1):8-17.

284. Litchfield J, Freeman J, Schou H, Elsley M, Fuller R, Chubb B. Is the future for clinical trials internetbased? A cluster randomized clinical trial. Clinical Trials. 2005;2(1):72-9.

285. Bjelland I, Dahl AA, Haug TT, Neckelmann D. The validity of the Hospital Anxiety and Depression Scale: An updated literature review. Journal of Psychosomatic Research. 2002;52(2):69-77.

286. Snaith RP. The Hospital Anxiety And Depression Scale. Health Qual Life Outcomes. 2003;1:29-.

287. Herrmann C. International experiences with the Hospital Anxiety and Depression Scale-A review of validation data and clinical results. Journal of Psychosomatic Research. 1997;42(1):17-41.

288. Hinz A, Brähler E. Normative values for the Hospital Anxiety and Depression Scale (HADS) in the general German population. Journal of Psychosomatic Research. 2011;71(2):74-8.

289. Singer S, Kuhnt S, Götze H, Hauss J, Hinz A, Liebmann A, et al. Hospital anxiety and depression scale cutoff scores for cancer patients in acute care. British Journal of Cancer. 2009;100(6):908-12.

290. Cosco TD, Doyle F, Ward M, McGee H. Latent structure of the Hospital Anxiety And Depression Scale: A 10-year systematic review. Journal of Psychosomatic Research. 2012;72(3):180-4.

291. Turk DC, Dworkin RH, Trudeau JJ, Benson C, Biondi DM, Katz NP, et al. Validation of the Hospital Anxiety and Depression Scale in Patients With Acute Low Back Pain. The Journal of Pain. 2015;16(10):1012-21.

292. Stasik-O'brien SM, Brock RL, Chmielewski M, Naragon-Gainey K, Koffel E, McDade-Montez E, et al. Clinical Utility of the Inventory of Depression and Anxiety Symptoms (IDAS). Assessment. 2019;26(5):944-60.

293. Brown TA, Barlow DH. A Proposal for a Dimensional Classification System Based on the Shared Features of the DSM-IV Anxiety and Mood Disorders: Implications for Assessment and Treatment. Psychological Assessment. 2009;21(3):256-71.

294. Rosellini AJ, Boettcher H, Brown TA, Barlow DH. A Transdiagnostic Temperament-Phenotype Profile Approach to Emotional Disorder Classification: An Update. Journal of experimental psychopathology. 2015;a2(1):110. 
295. Boettcher H, Correa J, Cassiello-Robbins C, Ametaj A, Rosellini AJ, Brown TA, et al. Dimensional Assessment of Emotional Disorder Outcomes in Transdiagnostic Treatment: A Clinical Case Study. Cognitive and Behavioral Practice. 2020.

296. McEvoy PM, Mahoney AEJ. Achieving certainty about the structure of intolerance of uncertainty in a treatment-seeking sample with anxiety and depression.(Report). Journal of Anxiety Disorders. 2011;25(1):112.

297. Carleton RN, Mulvogue MK, Thibodeau MA, McCabe RE, Antony MM, Asmundson GJG. Increasingly certain about uncertainty: Intolerance of uncertainty across anxiety and depression. Journal of Anxiety Disorders. 2012;26(3):468-79.

298. Hale W, Richmond M, Bennett J, Berzins T, Fields A, Weber D, et al. Resolving Uncertainty About the Intolerance of Uncertainty Scale-12: Application of Modern Psychometric Strategies. Journal of Personality Assessment. 2016;98(2):200-8.

299. Lauriola M, Mosca O, Carleton RN. Hierarchical factor structure of the intolerance of uncertainty scale short form (IUS-12) in the Italian version. TPM: Testing, Psychometrics, Methodology in Applied Psychology. 2016;23(3).

300. Shihata S, McEvoy PM, Mullan BA. A Bifactor Model of Intolerance of Uncertainty in Undergraduate and Clinical Samples: Do We Need to Reconsider the Two-Factor Model? Psychological Assessment. 2018;30(7):893-903.

301. Birrell J, Meares K, Wilkinson A, Freeston M. Toward a definition of intolerance of uncertainty: A review of factor analytical studies of the Intolerance of Uncertainty Scale. Clinical Psychology Review. 2011;31(7):1198-208.

302. Hong RY, Lee SSM. Further Clarifying Prospective and Inhibitory Intolerance of Uncertainty: Factorial and Construct Validity of Test Scores From the Intolerance of Uncertainty Scale. Psychological Assessment. 2015;27(2):605-20.

303. Fetzner M, Horswill S, Boelen P, Carleton R. Intolerance of Uncertainty and PTSD Symptoms: Exploring the Construct Relationship in a Community Sample with a Heterogeneous Trauma History. Cognitive Therapy and Research. 2013;37(4):725-34.

304. Helsen K, Van Den Bussche E, Vlaeyen JWS, Goubert L. Confirmatory factor analysis of the Dutch Intolerance of Uncertainty Scale: Comparison of the full and short version. Journal of Behavior Therapy and Experimental Psychiatry. 2013;44(1):21-9.

305. McEvoy PM, Mahoney AEJ. To Be Sure, To Be Sure: Intolerance of Uncertainty Mediates Symptoms of Various Anxiety Disorders and Depression. Behavior Therapy. 2012;43(3):533-45.

306. Khawaja NG, Yu LNH. A comparison of the 27-item and 12-item intolerance of uncertainty scales. Clinical Psychologist. 2010;14(3):97-106.

307. Jacoby RJ, Fabricant LE, Leonard RC, Riemann BC, Abramowitz JS. Just to be certain: Confirming the factor structure of the Intolerance of Uncertainty Scale in patients with obsessive-compulsive disorder. Journal of Anxiety Disorders. 2013;27(5):535-42. 
308. Boelen PA, Lenferink LIM. Latent class analysis of indicators of intolerance of uncertainty. Scandinavian Journal of Psychology. 2018;59(3):243-51.

309. Oglesby M, Allan N, Short N, Raines A, Schmidt N. Factor mixture modeling of intolerance of uncertainty. Psychological Assessment. 2017:435.

310. Correa KA, Liu H, Shankman SA. The role of intolerance of uncertainty in current and remitted internalizing and externalizing psychopathology. Journal of Anxiety Disorders. 2019;62:68-76.

311. Shihata S, McEvoy PM, Mullan BA, Carleton RN. Intolerance of uncertainty in emotional disorders: What uncertainties remain? Journal of Anxiety Disorders. 2016;41:115-24.

312. Nolen-Hoeksema S, Morrow J. A Prospective Study of Depression and Posttraumatic Stress Symptoms After a Natural Disaster: The 1989 Loma Prieta Earthquake. Journal of Personality and Social Psychology. 1991;61(1):115-21.

313. Meyer TJ, Miller ML, Metzger RL, Borkovec TD. Development and validation of the penn state worry questionnaire. Behaviour Research and Therapy. 1990;28(6):487-95.

314. McEvoy PM, Kingsep P. The post-event processing questionnaire in a clinical sample with social phobia. Behaviour Research and Therapy. 2006;44(11):1689-97.

315. Spinhoven P, van Hemert AM, Penninx BW. Repetitive negative thinking as a predictor of depression and anxiety: A longitudinal cohort study. Journal of Affective Disorders. 2018;241:216-25.

316. Kaplan DM, Palitsky R, Carey AL, Crane TE, Havens CM, Medrano MR, et al. Maladaptive repetitive thought as a transdiagnostic phenomenon and treatment target: An integrative review. Journal of Clinical Psychology. 2018;74(7):1126-36.

317. Corsi-Cabrera M, Galindo-Vilchis L, Del-Río-Portilla Y, Arce C, Ramos-Loyo J. Within-subject reliability and inter-session stability of EEG power and coherent activity in women evaluated monthly over nine months. Clin Neurophysiol. 2007;118(1):9-21.

318. van Diessen E, Numan T, van Dellen E, van Der Kooi AW, Boersma M, Hofman D, et al. Opportunities and methodological challenges in EEG and MEG resting state functional brain network research. Clin Neurophysiol. 2015;126(8):1468-81.

319. Team RC. R: A language and environment for statistical computing. Vienna, Austria2020.

320. Pascual-Marqui RD. Discrete, 3D distributed, linear imaging methods of electric neuronal activity. Part 1: exact, zero error localization. arXiv preprint arXiv:07103341. 2007.

321. Pascual-Marqui RD, Lehmann D, Koukkou M, Kochi K, Anderer P, Saletu B, et al. Assessing interactions in the brain with exact low-resolution electromagnetic tomography. Philosophical Transactions of the Royal Society A: Mathematical, Physical and Engineering Sciences. 2011;369(1952):3768-84.

322. Nichols TE, Holmes AP. Nonparametric permutation tests for functional neuroimaging: A primer with examples. Hum Brain Mapp. 2002;15(1):1-25.

323. Vollmer M, editor HRVTool-an Open-Source Matlab Toolbox for Analyzing Heart Rate Variability2019 2019: IEEE. 
324. Martínez CAG, Quintana AO, Vila XA, Touriño MJL, Rodríguez-Liñares L, Presedo JMR, et al. Heart rate variability analysis with the R package RHRV: Springer; 2017.

325. Ksander JC, Kark SM, Madan CR. Breathe Easy EDA: A MATLAB toolbox for psychophysiology data management, cleaning, and analysis. F1000Research. 2018;7.

326. Bach DR, Castegnetti G, Korn CW, Gerster S, Melinscak F, Moser T. Psychophysiological modeling: Current state and future directions. Psychophysiology. 2018;55(11):e13214.

327. Rosenbaum JF, Fava M, Hoog SL, Ascroft RC, Krebs WB. Selective serotonin reuptake inhibitor discontinuation syndrome: a randomized clinical trial. Biological Psychiatry. 1998;44(2):77-87.

328. Montgomery SA, Fava M, Padmanabhan SK, Guico-Pabia CJ, Tourian KA. Discontinuation symptoms and taper/poststudy-emergent adverse events with desvenlafaxine treatment for major depressive disorder. Int Clin Psychopharmacol. 2009;24(6):296-305.

329. Baldwin DS, Montgomery SA, Nil R, Lader M. Discontinuation symptoms in depression and anxiety disorders. International Journal of Neuropsychopharmacology. 2007;10(1):73-84.

330. Rogel A, Guez J, Getter N, Keha E, Cohen T, Amor T, et al. Transient Adverse Side Effects During Neurofeedback Training: A Randomized, Sham-Controlled, Double Blind Study. Appl Psychophysiol Biofeedback. 2015;40(3):209-18.

331. Insel TR, Wang PS. Rethinking mental illness. JAMA. 2010;303(19):1970-1.

\section{Tables}

\section{Table 1: Template for Intervention Description and Replication (TIDieR)}




\begin{tabular}{|c|c|c|}
\hline $\begin{array}{l}\text { Item } \\
\text { number }\end{array}$ & Item & Description \\
\hline 1 & $\begin{array}{l}\text { BRIEF NAME } \\
\text { Provide the name or } \\
\text { a phrase that } \\
\text { describes the } \\
\text { intervention. }\end{array}$ & $\begin{array}{l}\text { InfraSlow fluctuation neurofeedback for Anxiety \& } \\
\text { Depression (ISAD) trial }\end{array}$ \\
\hline 2 & $\begin{array}{l}\text { WHY } \\
\text { Describe any } \\
\text { rationale, theory, or } \\
\text { goal of the elements } \\
\text { essential to the } \\
\text { intervention. }\end{array}$ & $\begin{array}{l}\text { eISFs are believed to coordinate and integrate } \\
\text { information exchange within and between core-ICNs. } \\
\text { Further, communication within and between core-ICNs } \\
\text { has been found to be disrupted in ID populations. We } \\
\text { hypothesize that eISF-NFB targeting key cortical nodes } \\
\text { of these core-ICNs, may restore proper intra- and inter- } \\
\text { network function and reduce ID-related symptoms. }\end{array}$ \\
\hline 3 & $\begin{array}{l}\text { WHAT } \\
\text { Materials: Describe } \\
\text { any physical or } \\
\text { informational } \\
\text { materials used in } \\
\text { the intervention, } \\
\text { including those } \\
\text { provided to } \\
\text { participants or used } \\
\text { in intervention } \\
\text { delivery or training } \\
\text { of intervention } \\
\text { providers. Provide } \\
\text { information on } \\
\text { where the materials } \\
\text { can be accessed } \\
\text { (e.g. online } \\
\text { appendix, URL). }\end{array}$ & $\begin{array}{l}\text { 19-channel sLORETA eISF-NFB training will be } \\
\text { performed using a 21-channel DC coupled amplifier } \\
\text { (Brainmaster Technologies Inc.), ASUS laptop computer } \\
\text { (ASUSTek Computer Inc.; 64.0 GB RAM; Intel Core i7 } \\
\text { processor) running BrainAvatar software (version } \\
\text { 4.7.5.844), } 24 \text { (Ag/AgCl) electrode Comby EEG caps, } \\
\text { blunt needle, } 5 \mathrm{ml} \text { syringe, and electrolyte gel (Electro- } \\
\text { Cap International Inc.). Free, open-source software } \\
\text { (Audacity.com) is used to record/playback all auditory } \\
\text { rewards during active/sham sessions. }\end{array}$ \\
\hline 4 & $\begin{array}{l}\text { Procedures: } \\
\text { Describe each of the } \\
\text { procedures, } \\
\text { activities, and/or }\end{array}$ & $\begin{array}{l}\text { Subjects will be asked to arrive with non-braided, clean, } \\
\text { dry hair. They will be seated in a comfortable chair with } \\
\text { their eyes closed in a quiet, cool }\left(\sim 15^{\circ} \mathrm{C}\right) \text {, dimly lit room. } \\
\text { An appropriately sized Comby EEG cap will be affixed to }\end{array}$ \\
\hline
\end{tabular}




\begin{tabular}{|c|c|c|}
\hline & $\begin{array}{l}\text { processes used in } \\
\text { the intervention, } \\
\text { including any } \\
\text { enabling or support } \\
\text { activities. }\end{array}$ & $\begin{array}{l}\text { the head and, using a blunt need and syringe, the scalp } \\
\text { will be mildly abraded just prior to the application of the } \\
\text { electrolyte gel beneath each electrode. EEGs will be } \\
\text { recorded with the Ag/AgCl electrodes positioned } \\
\text { according to the International 10-20 system (i.e., Fp1, } \\
\text { Fp2, F3, F4, C3, C4, P3, P4, O1, O2, F7, F8, T3, T4, T5, } \\
\text { T6, Fz, Cz, Pz) using a linked mastoids reference and a } \\
\text { ground electrode positioned centrally between, F3, Fp1, } \\
\text { Fz and Fpz. Impedances will be kept below } 10 \mathrm{k} \Omega \text { and a } \\
50 \text { Hz notice filter will be set. } \\
\text { Immediately prior to each training period, a } \\
\text { demonstration of motion/EMG artefact alerts will be } \\
\text { provided with instructions to avoid eye/head/face } \\
\text { movements in order to minimize this non-rewarding } \\
\text { feedback. Participants will then be instructed to close } \\
\text { their eyes, relax, stay awake, and listen to the sound } \\
\text { being played. They will be informed that the sound they } \\
\text { hear reflects that they are doing well. } \\
\text { Continuous, real-time auditory feedback (organ tones) } \\
\text { will be used for reinforcement when the subject's eISFs } \\
\text { surpass the threshold(s). The reward threshold will be } \\
\text { manually adjusted in real-time to maintain a } 60 \% \pm 10 \% \\
\text { success rate. The yoked-sham sessions will be identical to } \\
\text { active sessions, including live EEG recordings and real- } \\
\text { time motion/EMG artefact alerts, however the auditory } \\
\text { rewards will derive from playbacks of consecutive, pre- } \\
\text { recorded sessions of another female with IDs. } \\
\text { The trainer will remain present for the duration of all } \\
\text { sessions to monitor the EEG. }\end{array}$ \\
\hline 5 & $\begin{array}{l}\text { WHO PROVIDED } \\
\text { For each category } \\
\text { of intervention } \\
\text { provider (e.g. } \\
\text { psychologist, } \\
\text { nursing assistant), } \\
\text { describe their } \\
\text { expertise, }\end{array}$ & $\begin{array}{l}\text { A unblinded doctoral student with } 2+\text { years of training } \\
\text { and experience in the administration of NFB }\end{array}$ \\
\hline
\end{tabular}




\begin{tabular}{|c|c|c|}
\hline & $\begin{array}{l}\text { background and any } \\
\text { specific training } \\
\text { given. }\end{array}$ & \\
\hline 6 & $\begin{array}{l}\text { HOW } \\
\text { Describe the modes } \\
\text { of delivery (e.g. } \\
\text { face-to-face or by } \\
\text { some other } \\
\text { mechanism, such as } \\
\text { internet or } \\
\text { telephone) of the } \\
\text { intervention and } \\
\text { whether it was } \\
\text { provided } \\
\text { individually or in a } \\
\text { group. }\end{array}$ & $\begin{array}{l}\text { eISF-NFB sessions will be performed one-on-one and } \\
\text { face-to-face }\end{array}$ \\
\hline 7 & $\begin{array}{l}\text { WHERE } \\
\text { Describe the } \\
\text { type(s) of } \\
\text { location(s) where } \\
\text { the intervention } \\
\text { occurred, including } \\
\text { any necessary } \\
\text { infrastructure or } \\
\text { relevant features. }\end{array}$ & $\begin{array}{l}\text { eISF-NFB sessions will take place in the EEG lab of the } \\
\text { Department of Psychological Medicine, University of } \\
\text { Otago, Dunedin, New Zealand. }\end{array}$ \\
\hline 8 & $\begin{array}{l}\text { WHEN and HOW } \\
\text { MUCH } \\
\text { Describe the } \\
\text { number of times the } \\
\text { intervention was } \\
\text { delivered and over } \\
\text { what period of time } \\
\text { including the } \\
\text { number of sessions, } \\
\text { their schedule, and } \\
\text { their duration, } \\
\text { intensity or dose. }\end{array}$ & $\begin{array}{l}\text { Participants will attend three } 30 \text {-minute sessions per } \\
\text { week over } 4 \text { consecutive weeks ( } 12 \text { sessions in total). }\end{array}$ \\
\hline
\end{tabular}




\begin{tabular}{|c|c|c|}
\hline 9 & $\begin{array}{l}\text { TAILORING } \\
\text { If the intervention } \\
\text { was planned to be } \\
\text { personalised, } \\
\text { titrated or adapted, } \\
\text { then describe what, } \\
\text { why, when, and } \\
\text { how. }\end{array}$ & $\begin{array}{l}\text { Auditory feedback during active eISF-NFB sessions will } \\
\text { be based on each individual's real-time EEG-derived } \\
\text { cortical eISFs. Thresholds will be manually adjusted, } \\
\text { as needed, to maintain the pre-specified feedback success } \\
\text { rate. }\end{array}$ \\
\hline 10 & $\begin{array}{l}\text { MODIFICATIONS } \\
\text { If the intervention } \\
\text { was modified during } \\
\text { the course of the } \\
\text { study, describe the } \\
\text { changes (what, why, } \\
\text { when, and how). }\end{array}$ & Not applicable. This is a protocol. \\
\hline 11 & $\begin{array}{l}\text { HOW WELL } \\
\text { Planned: If } \\
\text { intervention } \\
\text { adherence or } \\
\text { fidelity was } \\
\text { assessed, describe } \\
\text { how and by whom, } \\
\text { and if any strategies } \\
\text { were used to } \\
\text { maintain or improve } \\
\text { fidelity, describe } \\
\text { them. }\end{array}$ & $\begin{array}{l}\text { Protocol adherence will be monitored by the trainer. } \\
\text { Attempts will be made to mitigate adherence issues via } \\
\text { automated email and text message reminders sent on the } \\
\text { day of each training session. }\end{array}$ \\
\hline 12 & $\begin{array}{l}\text { Actual: If } \\
\text { intervention } \\
\text { adherence or } \\
\text { fidelity was } \\
\text { assessed, describe } \\
\text { the extent to which } \\
\text { the intervention was } \\
\text { delivered as } \\
\text { planned. }\end{array}$ & Not applicable. This is a protocol. \\
\hline
\end{tabular}


Figures



Figure 1 
The trial consists of a 1-week pre-intervention (i.e. baseline assessments) phase, 4-week intervention (i.e. sLORETA elSF-NFB) phase, and 4-week post-intervention (i.e. follow-up) phase. The total trial period will be approximately 9 -weeks

\section{Supplementary Files}

This is a list of supplementary files associated with this preprint. Click to download.

- AdditionalFile1MCCvoxels.csv

- AdditionalFile2PCCvoxels.csv 